\title{
A NEW CONSTRUCTION OF THE ASYMPTOTIC ALGEBRA ASSOCIATED TO THE $q$-SCHUR ALGEBRA
}

\author{
OLIVIER BRUNAT AND MAX NEUNHÖFFER
}

\begin{abstract}
We denote by $A$ the ring of Laurent polynomials in the indeterminate $v$ and by $K$ its field of fractions. In this paper, we are interested in representation theory of the "generic" $q$-Schur algebra $\mathcal{S}_{q}(n, r)$ over $A$. We will associate to every symmetrising trace form $\tau$ on $K \mathcal{S}_{q}(n, r)$ a subalgebra $\mathcal{J}_{\tau}$ of $K \mathcal{S}_{q}(n, r)$ which is isomorphic to the "asymptotic" algebra $\mathcal{J}(n, r)_{A}$ defined by J. Du. As a consequence, we give a new hypothesis which implies James' conjecture.
\end{abstract}

\section{INTRODUCTION}

This article is concerned with the representation theory of the "generic" $q$-Schur algebra $\mathcal{S}_{q}(n, r)$ over $A=\mathbb{Z}\left[v, v^{-1}\right]$. The $q$-Schur algebra was introduced by Dipper and James in [3] and [4. There is an interest in studying the representations of this algebra, because they relate information about the modular representation theory of the finite general linear group $\mathrm{GL}_{n}(q)$ and of the quantum groups.

Using a new basis of $\mathcal{S}_{q}(n, r)$ constructed in [5] (which is analogous to the Kazhdan-Lusztig basis in Iwahori-Hecke algebras), J. Du introduced in 7 the asymptotic algebra $\mathcal{J}(n, r)_{A}$ over $A$ and defined a homomorphism, $\Phi: \mathcal{S}_{q}(n, r) \rightarrow$ $\mathcal{J}(n, r)_{A}$, the so-called Du-Lusztig homomorphism because its construction is similar to the Lusztig homomorphism for Iwahori-Hecke algebras.

There is a relevant open question in the representation theory of the $q$-Schur algebra, the so-called James' conjecture. A precise formulation of this conjecture is recalled in Section 6. In [11] Meinolf Geck obtained a new formulation of this conjecture. More precisely, for $k$ any field of characteristic $\ell$ and for $R$ any integral domain with quotient field $k$, if $q \in R$ is invertible, we can define the corresponding $q$-Schur algebra $\mathcal{S}_{q}(n, r)_{R}$ over $R$ and its extension of scalars $\mathcal{S}_{q}(n, r)_{k}$. Similarly, we can define $\mathcal{J}(n, r)_{k}$.

In [1, 1.2] M. Geck has shown that James' conjecture holds if and only if, for $\ell>r$, the rank of the homomorphism $\Phi_{k}: \mathcal{S}_{q}(n, r)_{k} \rightarrow \mathcal{J}(n, r)_{k}$ only depends on the multiplicative order of $q$ in $k^{\times}$, but not on $\ell$.

Thus, in order to prove James' conjecture, it is relevant to understand the rank of the Du-Lusztig homomorphism. The motivation of this paper is to develop new methods allowing us to study this rank. More precisely, we will give a new construction of the asymptotic algebra. Indeed, thanks to methods developed in [16 by the second author and adapted to our situation, we prove that $\mathcal{J}(n, r)_{A}$ is isomorphic to an algebra $\mathcal{J}_{\tau}$, which only depends on the choice of a symmetrising

Received by the editors January 9, 2009 and, in revised form, April 2, 2010.

2010 Mathematics Subject Classification. Primary 20C08, 20F55; Secondary 20 G05.

(C)2012 American Mathematical Society

Reverts to public domain 28 years from publication 
trace form $\tau$ on the semisimple algebra $K \mathcal{S}_{q}(n, r)$ (here $K=\mathbb{Q}(v)$ ) such that

$$
\mathcal{S}_{q}(n, r) \subseteq \mathcal{J}_{\tau} \subseteq K \mathcal{S}_{q}(n, r) .
$$

Note that we include non-degeneracy in the definition of "symmetrising trace form". Our main tool is to use the structure of the left cell modules of $\mathcal{S}_{q}(n, r)$ to construct an explicit Wedderburn basis of $K \mathcal{S}_{q}(n, r)$ (see Theorem 4.12). The main result of this paper is Theorem 5.5 .

The article is organised as follows. In Section 2, we recall the definition of the "generic" $q$-Schur algebra and of its analogue of the Kazhdan-Lusztig basis for Iwahori-Hecke algebras. In Section 3 we prove that the $q$-Schur algebra satisfies properties which are very similar to Lusztig's conjectures P1, ., P15 for IwahoriHecke algebras. In Section 4 we develop some tools to prove our main result in Section 5. Finally, in Section 6 we state a new hypothesis which implies James' conjecture.

\section{The Iwahori-Hecke Algebra of type A And the $q$-Schur Algebra}

Let $v$ be an indeterminate. We set $A=\mathbb{Z}\left[v, v^{-1}\right]$ to be the ring of Laurent polynomials in $v$ and $K:=\mathbb{Q}(v)$ its field of fractions. In order to introduce the $q$-Schur algebra over $A$, we have to recall some definitions and properties about Iwahori-Hecke algebras. We follow [15].

2.1. Iwahori-Hecke algebras and the Kazhdan-Lusztig basis. Let $(W, S)$ be a Coxeter group (here $S$ is the set of simple reflections). We define the corresponding Iwahori-Hecke algebra $\mathcal{H}$ as the free $A$-module with basis $\left\{T_{w}\right\}_{w \in W}$ satisfying

$$
\begin{array}{ll}
T_{w} T_{w^{\prime}}=T_{w w^{\prime}} & \text { if } l\left(w w^{\prime}\right)=l(w)+l\left(w^{\prime}\right), \\
\left(T_{s}-v\right)\left(T_{s}+v^{-1}\right)=0 & \text { for } s \in S,
\end{array}
$$

where $l$ is the length function on $W$. In [14, §1] Kazhdan and Lusztig define an $A$-basis $\left\{C_{w} \mid w \in W\right\}$ of $\mathcal{H}$ which satisfies

$$
\bar{C}_{w}=C_{w} \quad \text { and } \quad C_{w}=\sum_{y \leq w} p_{y, w} T_{y} \quad \text { for } w \in W,
$$

where $\leq$ is the Bruhat-Chevalley order on $W$, and ${ }^{-}: \mathcal{H} \rightarrow \mathcal{H}$ is the involutive automorphism of $\mathcal{H}$ defined by $\bar{v}=v^{-1}$ and $\overline{\sum_{w \in W} a_{w} T_{w}}=\sum_{w \in W} \bar{a}_{w} T_{w^{-1}}^{-1}$ and $p_{y, w} \in\left\langle v^{k} \mid k \leq 0\right\rangle_{\mathbb{Z}}$ and $p_{w, w}=1$.

Note that we use the more modern notation from [15, that is, our elements $T_{w}$ here are the same as in [15] and were denoted by $v^{-l(w)} T_{w}$ in [14, and our elements $C_{w}$ here were denoted by $C_{w}^{\prime}$ in [14] and by $c_{w}$ in [15].

We denote by $g_{x, y, z}$ the structure constants of $\mathcal{H}$ with respect to the basis $\left\{C_{w} \mid w \in W\right\}$, that is, we have

$$
C_{x} C_{y}=\sum_{z \in W} g_{x, y, z} C_{z} \quad \text { for } x, y \in W .
$$

We define a relation $y \preccurlyeq_{L} w$ on $W$ by: either $y=w$ or there is an $s \in S$ such that $g_{s, w, y} \neq 0$. Let $\leq_{L}$ be the transitive closure of the relation $\preccurlyeq_{L}$ and denote by $\sim_{L}$ the associated equivalence relation on $W$. The classes for this relation are the so-called left cells. Similarly, we define $\leq_{R}$ and $\sim_{R}$, and we call the corresponding equivalence classes right cells. For $y, w \in W$, we write $y \leq_{L R} w$ if there is a sequence $y=y_{0}, y_{1}, \ldots, y_{n}=w$ of elements of $W$ such that, for $i \in\{0, \ldots, n-1\}$, 
we have $y_{i} \leq_{L} y_{i+1}$ or $y_{i} \leq_{R} y_{i+1}$. The classes of the equivalence relation $\sim_{L R}$ on $W$ corresponding to $\leq_{L R}$ are the so-called two-sided cells.

In [15, §3.6], Lusztig shows that for $z \in W$, there is a unique integer $\mathbf{a}(z)$ such that for every $x, y \in W$, we have $g_{x, y, z} \in v^{\mathbf{a}(z)} \mathbb{Z}\left[v^{-1}\right]$ and $g_{x, y, z} \notin v^{\mathbf{a}(z)-1} \mathbb{Z}\left[v^{-1}\right]$. Moreover, for $z \in W$, we define $\Delta(z)=-\operatorname{deg} p_{1, z}$. For $x, y, z \in W$, we write $\gamma_{x, y, z^{-1}} \in \mathbb{Z}$ for the coefficient of $v^{\mathbf{a}(z)}$ in $g_{x, y, z}$ and we set

$$
\mathcal{D}=\{d \in W \mid \mathbf{a}(d)=\Delta(d)\},
$$

the set of distinguished involutions. In the case that $W$ is a finite Weyl group, an affine Weyl group, or a dihedral group, Lusztig proved that the following conjectures hold (see [15, $\S \S 15-17]$ ):

P1 For any $z \in W$ we have $\mathbf{a}(z) \leq \Delta(z)$.

P2 Let $x, y \in W$; if $\gamma_{x, y, d} \neq 0$ for some $d \in \mathcal{D}$, then we have $x=y^{-1}$.

P3 If $y \in W$, there exists a unique $d \in \mathcal{D}$ such that $\gamma_{y^{-1}, y, d} \neq 0$.

$\mathbf{P} 4$ If $x \leq_{L R} y$, then $\mathbf{a}(x) \geq \mathbf{a}(y)$.

P5 If $d \in \mathcal{D}$ and $y \in W$ are such that $\gamma_{y^{-1}, y, d} \neq 0$, then $\gamma_{y^{-1}, y, d}= \pm 1$.

P6 For $d \in \mathcal{D}$, we have $d=d^{-1}$.

P7 For every $x, y, z \in W$, we have $\gamma_{x, y, z}=\gamma_{y, z, x}=\gamma_{z, x, y}$.

$\mathbf{P 8}$ Let $x, y, z \in W$ be such that $\gamma_{x, y, z} \neq 0$, then $x \sim_{L} y^{-1}, y \sim_{L} z^{-1}$ and $z \sim_{L} x^{-1}$.

P9 If $x \leq_{L} y$ and $\mathbf{a}(x)=\mathbf{a}(y)$, then $x \sim_{L} y$.

$\mathbf{P 1 0}$ If $x \leq_{R} y$ and $\mathbf{a}(x)=\mathbf{a}(y)$, then $x \sim_{R} y$.

$\mathbf{P 1 1}$ If $x \leq_{L R} y$ and $\mathbf{a}(x)=\mathbf{a}(y)$, then $x \sim_{L R} y$.

P13 Every left cell contains a unique element $d \in \mathcal{D}$ and $\gamma_{y^{-1}, y, d} \neq 0$ for every $y \sim_{L} d$.

P14 For every $x \in W$, we have $x \sim_{L R} x^{-1}$.

$\mathbf{P 1 5}$ Let $v^{\prime}$ be a second indeterminate and let $g_{x, y, z}^{\prime} \in \mathbb{Z}\left[v^{\prime}, v^{\prime-1}\right]$ be obtained from $g_{x, y, z}$ by the substitution $v \mapsto v^{\prime}$. If $x, x^{\prime}, y, w \in W$ satisfy $\mathbf{a}(w)=$ $\mathbf{a}(y)$, then

$$
\sum_{y^{\prime}} g_{w, x^{\prime}, y^{\prime}}^{\prime} g_{x, y^{\prime}, y}=\sum_{y^{\prime}} g_{x, w, y^{\prime}} g_{y^{\prime}, x^{\prime}, y}^{\prime} .
$$

Note that in this paper we only consider the case of type $\mathrm{A}$, in which $W$ is the symmetric group on $|S|+1$ points.

2.2. The $q$-Schur algebra $\mathcal{S}_{q}(n, r)$. In the following, we denote by $W$ the symmetric group of degree $r$, and by $S$ the set of transpositions $s_{i}=(i, i+1)$ for $1 \leq i \leq r-1$ and $\mathcal{H}$ is the associated Iwahori-Hecke algebra as in $\$ 2.1$. Let $n, r \geq 1$, we denote by $\Lambda(n, r)$ the set of compositions of $r$ into at most $n$ parts. For $\lambda \in \Lambda(n, r)$, we denote by $W_{\lambda} \subseteq W$ the corresponding Young subgroup. For $\lambda, \mu \in \Lambda(n, r)$, we set $D_{\lambda, \mu}$ to be the set of distinguished double coset representatives of $W$ with respect to $W_{\lambda}$ and $W_{\mu}$. We set

$$
M(n, r)=\left\{(\lambda, w, \mu) \mid \lambda, \mu \in \Lambda(n, r), w \in D_{\lambda, \mu}\right\} .
$$

For $\underline{a}=(\lambda, w, \mu) \in M(n, r)$, we write $r o(\underline{a})=\lambda$ and $\operatorname{co}(\underline{a})=\mu$ and we set $\underline{a}^{t}=$ $\left(\mu, w^{-1}, \lambda\right)$. For $\lambda, \mu \in \Lambda(n, r)$, we set $M_{\lambda, \mu}=\{\underline{a} \in M(n, r) \mid \operatorname{ro}(\underline{a})=\lambda, \operatorname{co}(\underline{a})=$ $\mu\}$. We remark that if $w \in D_{\lambda, \mu}$, then the double coset $W_{\lambda} w W_{\mu}$ has a unique longest element. To prove this, we can proceed as follows: We denote by $w_{0}$ the longest element of $W$, then ${ }^{w_{0}} W_{\mu}=W_{\widetilde{\mu}}$. Here $\widetilde{\mu}=\left(\mu_{s}, \mu_{s-1}, \ldots, \mu_{1}\right)$, where $\mu=\left(\mu_{1}, \ldots, \mu_{s}\right)$. Moreover, $r_{w_{0}}: W \rightarrow W, x \mapsto x w_{0}$ induces a bijection from the 
double coset $W_{\lambda} w w_{0} W_{\widetilde{\mu}}$ to the double coset $W_{\lambda} w W_{\mu}$. Thanks to [15, 11.3], we deduce that $r_{w_{0}}$ reverses the Bruhat order. Since the double coset $W_{\lambda} w w_{0} W_{\widetilde{\mu}}$ has a unique element of minimal length, the result follows. We write $D_{\lambda, \mu}^{+}$for the set of double coset representatives of maximal length. We denote by $\ell_{\lambda, \mu}$ the bijection from $D_{\lambda, \mu}$ to $D_{\lambda, \mu}^{+}$that associates to the representative of minimal length $w$ of the double coset $W_{\lambda} w W_{\mu}$ the representative of maximal length. We remark that if $w \in D_{\lambda, \mu}$, then $w^{-1} \in D_{\mu, \lambda}$. Moreover, we have

$$
\ell_{\lambda, \mu}(w)^{-1}=\ell_{\mu, \lambda}\left(w^{-1}\right) .
$$

In the following, we set $\sigma(\underline{a}):=\ell_{\lambda, \mu}(w)$ for $\underline{a}=(\lambda, w, \mu)$.

We now recall the definition of the $q$-Schur algebra $\mathcal{S}_{q}(n, r)$ introduced by Dipper and James in [3]. We set $q=v^{2}$, then the $q$-Schur algebra $\mathcal{S}_{q}(n, r)$ of degree $(n, r)$ is the endomorphism algebra

$$
\mathcal{S}_{q}(n, r)=\operatorname{End}_{\mathcal{H}}\left(\bigoplus_{\lambda \in \Lambda(n, r)} x_{\lambda} \mathcal{H}\right),
$$

where $x_{\lambda}=\sum_{w \in W_{\lambda}} v^{l(w)} T_{w} \in \mathcal{H}$. In [2, 3.4] Dipper and James prove that $\mathcal{S}_{q}(n, r)$ has a standard basis $\left\{\phi_{\lambda, \mu}^{w} \mid(\lambda, w, \mu) \in M(n, r)\right\}$ indexed by the set $M(n, r)$, which plays the same role as the basis $\left\{T_{w} \mid w \in W\right\}$ for the Iwahori-Hecke algebra $\mathcal{H}$. Moreover, in [5] Du proves that $\mathcal{S}_{q}(n, r)$ has another basis $\left\{\theta_{\underline{a}} \mid \underline{a} \in M(n, r)\right\}$ whose construction is analogous to the Kazhdan-Lusztig basis of $\mathcal{H}$. We denote by $f_{\underline{a}, \underline{b}, \underline{c}} \in A$ the structure constants with respect to this basis, that is, we have

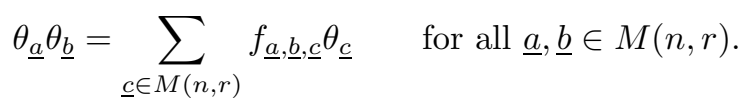

We recall the following lemma:

Lemma 2.3. We have $f_{\underline{a}, \underline{b}, \underline{c} \neq} \neq 0$ only if $\operatorname{co}(\underline{a})=\operatorname{ro}(\underline{b})$ and $(\operatorname{ro}(\underline{a}), \operatorname{co}(\underline{b}))=$ $(\operatorname{ro}(\underline{c}), c o(\underline{c}))$. In this case, we have

$$
f_{\underline{a}, \underline{b}, \underline{c}}=h_{\mu}^{-1} g_{\sigma(\underline{a}), \sigma(\underline{b}), \sigma(\underline{c})} .
$$

where $\mu=c o(\underline{a})=r o(\underline{b})$ and $h_{\mu}=\sum_{w \in W_{\mu}} v^{2 l(w)-l\left(w_{\mu}\right)}$ (here $w_{\mu}$ denotes the longest element in $\left.W_{\mu}\right)$ and $g_{\sigma(\underline{a}), \sigma(\underline{b}), \sigma(\underline{c})}$ is the structure constant of $\mathcal{H}$ defined in Section 2.1.

Proof. See [5, Prop. 3.4]. We want to explain why we have a further hypothesis here than in [5, Prop. 3.4]: For $\underline{a}=(\lambda, w, \mu) \in M(n, r)$ the element $\theta_{\underline{a}}$ is by definition a linear combination of basis elements $\phi_{\lambda, \mu}^{z}$ for $z \in \mathcal{D}_{\lambda, \mu}$. Thus, viewed as endomorphism of $\bigoplus_{\lambda \in \Lambda(n, r)} x_{\lambda} \mathcal{H}$ it vanishes on all summands except on $x_{\mu} \mathcal{H}$ and maps into the summand $x_{\lambda} \mathcal{H}$. Thus, if either $\operatorname{co}(\underline{a}) \neq \operatorname{ro}(\underline{b})$ or $(\operatorname{ro}(\underline{a}), \operatorname{co}(\underline{b})) \neq$ $(r o(\underline{c}), c o(\underline{c}))$, the structure constant $f_{\underline{a}, \underline{b}, \underline{c}}$ vanishes also. If both equations hold, the proof in [5, Prop. 3.4] works using $g_{\sigma(\underline{a}), \sigma(\underline{b}), \sigma(\underline{c})}$.

We are not claiming that [5, Prop. 3.4] is wrong as stated there. However, the notation $g_{\underline{a}, \underline{b}, \underline{c}}$ there needs proper interpretation (see [5, Section 3.3]), a problem we avoid here.

Remark 2.4. To further explain the just mentioned change of notation, consider the following: Let $n=r=3, \lambda:=(2,1,0), \mu:=(1,1,1)$, and $\nu:=(2,1,0)$. Then $W$ is the symmetric group on 3 letters, generated by the two Coxeter generators $s_{1}=$ 
$(1,2)$ and $s_{2}=(2,3)$. Thus $\mathcal{D}_{\lambda, \mu}^{+}:=\left\{s_{1}, s_{1} s_{2}, s_{1} s_{2} s_{1}\right\}, \mathcal{D}_{\mu, \nu}^{+}=\left\{s_{1}, s_{2} s_{1}, s_{1} s_{2} s_{1}\right\}$ and $\mathcal{D}_{\lambda, \nu}^{+}=\left\{s_{1}, s_{1} s_{2} s_{1}\right\}$.

By the relations, we have $T_{s_{1}} \cdot T_{s_{2} s_{1}}=T_{s_{1} s_{2} s_{1}}$ and thus $g_{s_{1}, s_{2} s_{1}, s_{1} s_{2} s_{1}}=1$. We now set $\underline{a}:=(\lambda, \mathrm{id}, \mu), \underline{b}:=\left(\mu, s_{2}, \nu\right)$ and $\underline{c}:=\left(\lambda, s_{2}, \nu\right)$. Thus, we get

$$
f_{\underline{a}, \underline{b}, \underline{c}}=1 \cdot g_{\sigma(\underline{a}), \sigma(\underline{b}), \sigma(\underline{c})}=g_{s_{1}, s_{2} s_{1}, s_{1} s_{2} s_{1}}=1,
$$

since here $h_{\mu}=1$.

However, if we set $\underline{a^{\prime}}:=\left(\mu, s_{1}, \mu\right)$, then $f_{a^{\prime}, \underline{b}, c}=0$, because of $\operatorname{ro}\left(\underline{a^{\prime}}\right) \neq \operatorname{ro}(\underline{c})$ and the arguments in the proof of Lemma 2.3. On the other hand, we have $\operatorname{ro}\left(\underline{a}^{\prime}\right)=c o(\underline{b})$ and $g_{\sigma\left(\underline{a^{\prime}}\right), \sigma(\underline{b}), \sigma(\underline{c})}=g_{s_{1}, s_{2} s_{1}, s_{1} s_{2} s_{1}}=1$. This shows that we indeed need all of the hypotheses in Lemma 2.3. The statement in [5, Prop. 3.4] is true if one interprets $g_{\underline{a^{\prime}}, \underline{b}, \underline{c}}$ to be zero.

Definition 2.5 (The a-function and the distinguished elements). Following [7, Section 2], we extend the $\mathbf{a}$-function to $M(n, r)$ by setting $\mathbf{a}(\underline{a})=\mathbf{a}(\sigma(\underline{a}))$ for every $\underline{a} \in M(n, r)$ and we extend the set $\mathcal{D}$ to the set

$$
\mathcal{D}(n, r)=\{\underline{d} \in M(n, r) \mid \operatorname{co}(\underline{d})=r o(\underline{d}), \sigma(\underline{d}) \in \mathcal{D}\} .
$$

Moreover, for every $\underline{a}, \underline{b}, \underline{c} \in M(n, r)$, we define

$$
\gamma_{\underline{a}, \underline{b}, \underline{c}^{t}}= \begin{cases}\gamma_{\sigma(\underline{a}), \sigma(\underline{b}), \sigma\left(\underline{c}^{t}\right)}=\gamma_{\sigma(\underline{a}), \sigma(\underline{b}), \sigma(\underline{c})^{-1}} & \text { if } f_{\underline{a}, \underline{b}, \underline{c} \neq} \neq 0 \\ 0 & \text { otherwise }\end{cases}
$$

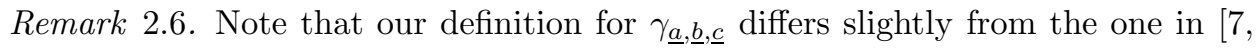

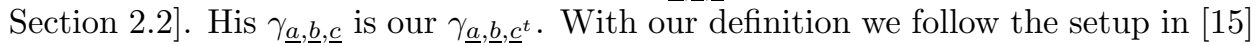
more closely and get nicer cyclic symmetries in our formulas.

Remark 2.7. In comparison to [7, Section 2.1] we added the explicit hypothesis for the elements $\underline{d} \in \mathcal{D}(n, r)$ that $r o(\underline{d})=c o(\underline{d})$. However, this hypothesis is implicit in [7, since otherwise the statements in [7, 4.1,(a)-(d)] and some others would not be true.

Now, for $\underline{a}, \underline{b} \in M(n, r)$, if there is $\underline{c} \in M(n, r)$ such that $f_{\underline{c}, \underline{b}, \underline{a}} \neq 0$, then we write $\underline{a} \leq_{L} \underline{b}$. We define $\leq_{R}$ by $\underline{a} \leq_{R} \underline{b}$ if and only if $\underline{a}^{t} \leq_{L} \underline{b}^{t}$. Moreover, we define $\leq_{L R}$ as in the Iwahori-Hecke algebra case. These relations induce corresponding equivalence relations $\sim_{L}, \sim_{R}$ and $\sim_{L R}$. We call the corresponding equivalence classes the left, right and two-sided cells of $M(n, r)$, respectively.

Let $\Gamma$ be a left cell of $M(n, r)$. We set

$$
\mathcal{S}_{\leq \Gamma}=\sum_{\underline{b} \leq_{L} \underline{a}} A \theta_{\underline{b}} \quad \text { and } \quad \mathcal{S}_{<\Gamma}=\sum_{\underline{b} \leq L \underline{a}, \underline{b} \varkappa_{L} \underline{a}} A \theta_{\underline{b}},
$$

for some $\underline{a} \in \Gamma$, both are clearly left ideals of $\mathcal{S}_{q}(n, r)$ by the definition of $\leq_{L}$. Then the left cell module $\mathrm{LC}^{(\Gamma)}$ corresponding to $\Gamma$ is defined as the quotient $\mathcal{S}_{\leq \Gamma} / \mathcal{S}_{<\Gamma}$.

We define the right cell module $\mathrm{RC}^{(\Gamma)}$ corresponding to a right cell $\Gamma$ of $M(n, r)$ similarly. To see that we get right ideals we have to use Lemma 2.3 and $g_{x, y, z}=$ $g_{y^{-1}, x^{-1}, z^{-1}}$ for $x, y, z \in W$ (see [15, 13.2.(e)]) together with $\sigma\left(\underline{a}^{t}\right)=\sigma(\underline{a})^{-1}$. This implies $f_{\underline{a}, \underline{b}, \underline{c}}=0$ if and only if $f_{\underline{b}^{t}, \underline{a}^{t}, \underline{c}^{t}}=0$. 


\section{Lusztig's CONJECtures FOR THE $q$-Schur ALGEBRA}

In this section, we prove that the $q$-Schur algebra satisfies properties very similar to $\mathbf{P 1}, \ldots, \mathbf{P 1 5}$ for the Iwahori-Hecke algebra. First, we give some preliminary results.

Lemma 3.1. If $\underline{a} \leq_{L} \underline{b}$ (resp. $\left.\leq_{R}, \leq_{L R}\right)$, then $\sigma(\underline{a}) \leq_{L} \sigma(\underline{b})$ (resp. $\leq_{R}$, $\left.\leq_{L R}\right)$.

Proof. Since $\underline{a} \leq_{L} \underline{b}$, there is $\underline{c} \in M(n, r)$ such that $f_{\underline{c}, \underline{b}, \underline{a}} \neq 0$; but we have $f_{\underline{c}, \underline{b}, \underline{a}}=$

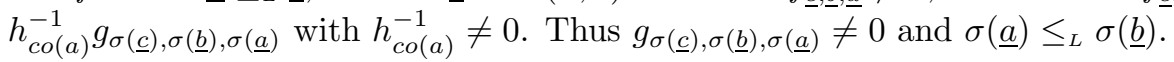

Lemma 3.2. If $\underline{a} \leq_{L} \underline{b}$, then $\operatorname{co}(\underline{a})=c o(\underline{b})$. If $\underline{a} \leq_{R} \underline{b}$, then $\operatorname{ro}(\underline{a})=\operatorname{ro}(\underline{b})$.

Proof. Since $\underline{a} \leq_{L} \underline{b}$ there is $\underline{c} \in M(n, r)$ such that $f_{\underline{c}, \underline{b}, \underline{a}} \neq 0$. From Lemma 2.3 it follows that $(\operatorname{ro}(\underline{a}), c o(\underline{a}))=(r o(\underline{c}), c o(\underline{b}))$ and the result is proved.

Lemma 3.3. Let $\lambda, \mu, \nu \in \Lambda(n, r), x \in D_{\lambda, \mu}^{+}$and $y \in D_{\mu, \nu}^{+}$. If $g_{x, y, z} \neq 0$ for some $z \in W$, then $z \in D_{\lambda, \nu}^{+}$.

Proof. For $\lambda \in \Lambda(n, r)$ we set $S_{\lambda}:=W_{\lambda} \cap S$, the set of Coxeter generators of the parabolic subgroup $W_{\lambda}$. Let $x \in \mathcal{D}_{\lambda, \mu}^{+}$and $y \in \mathcal{D}_{\mu, \nu}^{+}$and $g_{x, y, z} \neq 0$. On one hand, this means that $l(s x)<l(x)$ for all $s \in S_{\lambda}$ and $l(y s)<l(y)$ for all $s \in S_{\nu}$. On the other hand, we get $z \leq_{L} y$ and $z \leq_{R} x$ and thus $l(z s)<l(z)$ for all $s \in S$ with $l(y s)<l(y)$ and $l(s z)<l(s)$ for all $s \in S$ with $l(s x)<l(x)$ by [15. Lemma 8.6]. Thus we have, in particular, that $l(z s)<l(z)$ for all $s \in S_{\nu}$ and $l(s z)<l(z)$ for all $s \in S_{\lambda}$. Hence $z$ is the longest element in its $W_{\lambda}-W_{\nu}$-double coset in $W$.

Lemma 3.4. We have $\underline{a} \leq_{R} \underline{b}$ if and only if there is a $\underline{c} \in M(n, r)$ with $f_{\underline{b}, \underline{c}, \underline{a}} \neq 0$.

Proof. By definition, $\underline{a} \leq_{R} \underline{b}$ is equivalent to $\underline{a}^{t} \leq_{L} \underline{b}^{t}$. This in turn means that there is a $\underline{c} \in M(n, r)$ such that $f_{\underline{c}^{t}, \underline{b}^{t}, \underline{a}^{t}} \neq 0$. As mentioned at the end of Section 2.2 we have $f_{\underline{b}, \underline{c}, \underline{a}}=0$ if and only if $f_{\underline{c}^{t}, \underline{b}^{t}, \underline{a}^{t}}=0$ which directly implies the statement in the lemma.

We now recall the following result of $\mathrm{Du}[8,2.2,2.3]$.

Lemma 3.5. For any $\underline{a}, \underline{b} \in M(n, r)$, we have

(1) $\underline{a} \sim_{L R} \underline{b} \Longleftrightarrow \sigma(\underline{a}) \sim_{L R} \sigma(\underline{b})$.

(2) $\underline{a} \sim_{L} \underline{b} \Longleftrightarrow c o(\underline{a})=c o(\underline{b})$ and $\sigma(\underline{a}) \sim_{L} \sigma(\underline{b})$.

Remark 3.6. Note that to prove this result, the set $M(n, r)$ is identified with the set of $n \times n$ matrices whose entry sum is $r$. If $\left(a_{i j}\right)_{1 \leq i, j \leq n}$ is the matrix corresponding to $\underline{a} \in M(n, r)$, then $c o(\underline{a})=\left(\sum_{i} a_{i 1}, \ldots, \sum_{i} a_{i n}\right)$ and $r o(\underline{a})=\left(\sum_{i} a_{1 i}, \ldots, \sum_{i} a_{n i}\right)$; see [8, §2] for more details. 
Proposition 3.7. The following properties hold for the q-Schur algebra:

Q1 For any $\underline{a} \in M(n, r)$ we have $\mathbf{a}(\underline{a}) \leq \Delta(\sigma(\underline{a}))$.

Q2 If $\gamma_{a}, \underline{b}, \underline{d} \neq 0$ for some $\underline{d} \in \mathcal{D}(n, r)$, then we have $\underline{b}=\underline{a}^{t}$.

Q3 For every $\underline{a} \in M(n, r)$, there is a unique $\underline{d} \in \mathcal{D}(n, r)$ with $\gamma_{\underline{a}^{t}, \underline{a}, \underline{d}} \neq 0$.

Q4 If $\underline{a} \leq_{L R} \underline{b}$, then $\mathbf{a}(\underline{a}) \geq \mathbf{a}(\underline{b})$.

Q5 If $\underline{d} \in \mathcal{D}(n, r)$ and $\underline{a} \in M(n, r)$ are such that $\gamma_{\underline{a}^{t}, \underline{a}, \underline{d}} \neq 0$, then $\gamma_{\underline{a}^{t}, \underline{a}, \underline{d}}=1$.

Q6 For $\underline{d} \in \mathcal{D}(n, r)$, we have $\underline{d}=\underline{d}^{t}$.

Q7 For every $\underline{a}, \underline{b}, \underline{c} \in M(n, r)$, we have $\gamma_{\underline{a}, \underline{b}, \underline{c}}=\gamma_{\underline{b}, \underline{c}, \underline{a}}=\gamma_{\underline{c}, \underline{a}, \underline{b}}$.

Q8 Let $\underline{a}, \underline{b}, \underline{c} \in M(n, r)$ be such that $\gamma_{\underline{a}, \underline{b}, \underline{c}} \neq 0$, then $\underline{a} \sim_{L} \underline{b}^{t}, \underline{b} \sim_{L} \underline{c}^{t}$ and $\underline{c} \sim_{L} \underline{a}^{t}$.

$\mathbf{Q 9}$ If $\underline{a} \leq_{L} \underline{b}$ and $\mathbf{a}(\underline{a})=\mathbf{a}(\underline{b})$, then $\underline{a} \sim_{L} \underline{b}$.

$\mathbf{Q 1 0}$ If $\underline{a} \leq_{R} \underline{b}$ and $\mathbf{a}(\underline{a})=\mathbf{a}(\underline{b})$, then $\underline{a} \sim_{R} \underline{b}$.

Q11 If $\underline{a} \leq_{L R} \underline{b}$ and $\mathbf{a}(\underline{a})=\mathbf{a}(\underline{b})$, then $\underline{a} \sim_{L R} \underline{b}$.

Q13 Every left cell contains a unique element $\underline{d} \in \mathcal{D}(n, r)$ and $\gamma_{\underline{a}^{t}, \underline{a}, \underline{d}} \neq 0$

for every $\underline{a} \sim_{L} \underline{d}$.

Q14 For every $\underline{a} \in \bar{M}(n, r)$, we have $\underline{a} \sim_{L R} \underline{a}^{t}$.

$\mathbf{Q 1 5}$ Let $v^{\prime}$ be a second indeterminate and let $f_{x, y, z}^{\prime} \in \mathbb{Z}\left[v^{\prime}, v^{-1}\right]$ be obtained from $f_{x, y, z}$ by the substitution $v \mapsto v^{\prime}$. If $\underline{a}, \underline{a^{\prime}}, \underline{b}, \underline{c} \in W$ satisfy $\mathbf{a}(\underline{c})=$ $\mathbf{a}(\underline{b})$, then

$$
\sum_{\underline{b}^{\prime}} f_{\underline{c}, \underline{a}^{\prime}, \underline{b}^{\prime}}^{\prime} f_{\underline{a}, \underline{b}^{\prime}, \underline{b}}=\sum_{\underline{b}^{\prime}} f_{\underline{a}, \underline{c}, \underline{b^{\prime}}} f_{\underline{b}^{\prime}, \underline{a}^{\prime}, \underline{b}}^{\prime} .
$$

Proof. We note that $\mathbf{Q} \mathbf{1}$ is a direct consequence of Property $\mathbf{P} 1$.

We now will prove Property Q2. We suppose that $\gamma_{\underline{a}, \underline{b}, \underline{d}} \neq 0$ for some $\underline{a}, \underline{b} \in$ $M(n, r)$ and $\underline{d} \in \mathcal{D}(n, r)$. Since $\gamma_{\underline{a}, \underline{b}, \underline{d}} \neq 0$, it follows that $f_{\underline{a}, \underline{b}, \underline{d}} \neq 0$. Thus we have $c o(\underline{a})=r o(\underline{b}), r o(\underline{a})=r o(\underline{d})$ and $c o(\underline{b})=c o(\underline{d})$ by Lemma $[2.3$, but $c o(\underline{d})=r o(\underline{d})$ implies $r o(\underline{a})=c o(\underline{b})$. We now write $\underline{a}=\left(\lambda, w_{a}, \mu\right)$ and $\underline{b}=\left(\mu, w_{b}, \lambda\right)$. We have $\gamma_{\underline{a}, \underline{b}, \underline{d}}=\gamma_{\sigma(\underline{a}), \sigma(\underline{b}), \sigma(\underline{d})}$. From $\sigma(\underline{d}) \in \mathcal{D}$ we deduce using $\mathbf{P 2}$ that $\sigma(\underline{a})=\sigma(\underline{b})^{-1}$. It follows that $\ell_{\lambda, \mu}\left(w_{a}\right)=\ell_{\mu, \lambda}\left(w_{b}\right)^{-1}=\ell_{\lambda, \mu}\left(w_{b}^{-1}\right)$, we get $w_{a}=w_{b}^{-1}$ and thus Q2 holds.

Let $\underline{a}=(\lambda, w, \mu) \in M(n, r)$. Thanks to Property $\mathbf{P 3}$, there is a unique $d \in \mathcal{D}$ such that $\gamma_{\sigma(\underline{a})^{-1}, \sigma(\underline{a}), d} \neq 0$. Since $\sigma(\underline{a})^{-1}=\sigma\left(\underline{a}^{t}\right)$, we deduce that $g_{\sigma\left(\underline{a}^{t}\right), \sigma(\underline{a}), d} \neq 0$; but $\sigma\left(\underline{a}^{t}\right) \in D_{\mu, \lambda}^{+}$and $\sigma(\underline{a}) \in D_{\lambda, \mu}^{+}$, then Lemma 3.3 gives $d \in D_{\mu, \mu}^{+}$. We denote by $\widetilde{d}$ the representative of minimal length of the coset $W_{\mu} d W_{\mu}$ and we set $\underline{d}:=(\mu, \widetilde{d}, \mu)$. Then $\underline{d} \in \mathcal{D}(n, r)$ and $\sigma(\underline{d})=d$. It follows that $\gamma_{\underline{a}^{t}, \underline{a}, \underline{d}} \neq 0$ and thus $\mathbf{Q} 3$ holds.

Property Q4 follows from P4 and Lemma 3.1. Property Q5 directly follows from $\mathbf{P 5}$, since in our case $W$ is of type A and thus all coefficients of all KazhdanLusztig polynomials are non-negative by [15, 15.1].

Let $\underline{d}=(\lambda, w, \lambda) \in \mathcal{D}(n, r)$; we have $\sigma(\underline{d}) \in \mathcal{D}$, thus $\mathbf{P 6}$ gives $\sigma(\underline{d})^{-1}=\sigma(\underline{d})$. Therefore, we have $\ell_{\lambda, \lambda}(w)=\sigma(\underline{d})^{-1}=\sigma\left(\underline{d}^{t}\right)=\ell_{\lambda, \lambda}\left(w^{-1}\right)$, and it follows that $w=w^{-1}$; thus Q6 holds. Property Q7 follows directly from $\mathbf{P 7}$.

Suppose that $\gamma_{\underline{a}, \underline{b}, \underline{c}} \neq 0$ for some $\underline{a}, \underline{b}, \underline{c} \in M(n, r)$, then $f_{\underline{a}, \underline{b}, \underline{c} \underline{c}^{t}} \neq 0$ and it follows that $c o(\underline{a})=r o(\underline{b})$ and $(r o(\underline{a}), c o(\underline{b}))=\left(r o\left(\underline{c}^{t}\right), c o\left(\underline{c}^{t}\right)\right)$. Then we have

$$
\begin{aligned}
f_{\underline{b}^{t}, \underline{a}^{t}, \underline{c}} & =h_{c o(\underline{a})} g_{\sigma\left(\underline{b}^{t}\right), \sigma\left(\underline{a}^{t}\right), \sigma(\underline{c})} \\
& =h_{c o(\underline{a})} g_{\sigma(\underline{b})^{-1}, \sigma(\underline{a})^{-1}, \sigma(\underline{c})} \\
& =h_{c o(\underline{a})} g_{\sigma(\underline{a}), \sigma(\underline{b}), \sigma(\underline{c})^{-1}} \\
& =f_{\underline{a}, \underline{b}, \underline{c}^{t}} .
\end{aligned}
$$


It follows that $\underline{c}^{t} \leq_{L} \underline{b}$ and $\underline{c} \leq_{L} \underline{a}^{t}$. Using Q7 and the same arguments applied to $\gamma_{\underline{b}, \underline{c}, \underline{a}}=\gamma_{c, \underline{a}, \underline{b}} \neq 0$, we deduce that $\underline{a} \sim_{L} \underline{b}^{t}, \underline{b} \sim_{L} \underline{c}^{t}$ and $\underline{c} \sim_{L} \underline{a}^{t}$. Thus Q8 holds.

Next we prove Q13. Let $\underline{a} \in M(n, r)$. By Q3 there is a unique $\underline{d} \in \mathcal{D}(n, r)$ with $\gamma_{\underline{a}^{t}, \underline{a}, \underline{d}} \neq 0$ and for this $\underline{d}$ holds $\underline{a} \sim_{L} \underline{d}$ by Q8. But for $\underline{d}, \underline{d}^{\prime} \in \mathcal{D}(n, r)$ with $\underline{d} \sim_{L} \underline{d}^{\prime}$ we conclude $\operatorname{ro}(\underline{d})=\operatorname{co}(\underline{d})=\operatorname{co}\left(\underline{d}^{\prime}\right)=\operatorname{ro}\left(\underline{d}^{\prime}\right)$ using Lemma 3.2 and $\sigma(\underline{d})=\sigma\left(\underline{d}^{\prime}\right)$ using P13 since $\sigma(\underline{d}) \sim_{L} \sigma\left(\underline{d}^{\prime}\right)$ because of Lemma 3.1. Thus we have proved Q13.

Now, we prove Q9. Let $\underline{a}, \underline{b} \in M(n, r)$ with $\underline{a} \leq_{L} \underline{b}$ and $\mathbf{a}(\underline{a})=\mathbf{a}(\underline{b})$. By Lemma 3.1, we have $\sigma(\underline{a}) \leq_{L} \sigma(\underline{b})$. Hence, Property P9 implies that $\sigma(\underline{a}) \sim_{L} \sigma(\underline{b})$. Moreover, by Lemma 3.2, $c o(\underline{a})=c o(\underline{b})$. We then conclude with Lemma 3.5. The proof of Property Q11 is similar. Property Q10 follows from Q9 by transposition since $\mathbf{a}(\underline{a})=\mathbf{a}\left(\underline{a}^{t}\right)$ for all $\underline{a} \in M(n, r)$ (use [15, 13.9 (a)]).

Let $\underline{a} \in M(n, r)$ and $\underline{d} \in \mathcal{D}(n, r)$ be the unique element such that $\underline{a} \sim_{L} \underline{d}$ given by Q13. Then $\underline{a}^{t} \sim_{R} \underline{d}^{t}=\underline{d}$ and Q14 holds.

Finally, we prove Q15. We first remark that $f_{\underline{c}, \underline{a}^{\prime}, \underline{b}^{\prime}}^{\prime} \neq 0$ if and only if $f_{\underline{a}, \underline{c}, \underline{b^{\prime}}} \neq 0$, and $f_{\underline{a}, \underline{b}^{\prime}, \underline{b}} \neq 0$ if and only if $f_{\underline{b}^{\prime}, \underline{a}^{\prime}, \underline{b}}^{\prime} \neq 0$. Moreover, if $f_{\underline{c}, \underline{a}^{\prime}, \underline{b}^{\prime}}^{\prime} \neq 0$, then $f_{\underline{c}, \underline{a}^{\prime}, \underline{b}^{\prime}}^{\prime}=$ $h_{r o\left(\underline{a}^{\prime}\right)}^{\prime} g_{\sigma(\underline{c}), \sigma\left(\underline{a}^{\prime}\right), \sigma\left(\underline{b}^{\prime}\right)}$ and $f_{\underline{a}, \underline{c}, \underline{b}^{\prime}}=\underline{h}_{c o(\underline{a})} g_{\sigma(\underline{a}), \sigma(\underline{c}), \sigma\left(\underline{b}^{\prime}\right)}$. If $\underline{\underline{a}}_{\underline{a}, \underline{c}, \underline{b}^{\prime}} \neq 0$, then $f_{\underline{a}, \underline{c}, \underline{b^{\prime}}}=$ $h_{c o(\underline{a})} g_{\sigma(\underline{a}), \sigma(\underline{c}), \sigma\left(\underline{b}^{\prime}\right)}$ and $f_{\underline{b}^{\prime}, \underline{a}^{\prime}, \underline{b}}^{\prime}=h_{r o\left(\underline{a}^{\prime}\right)}^{\prime} g_{\sigma\left(\underline{b}^{\prime}\right), \sigma\left(\underline{a}^{\prime}\right), \sigma(\underline{b})}$. Here $h_{\mu}^{\prime}$ is obtained from $h_{\mu}$ by the substitution $v \mapsto v^{\prime}$. We note that $h_{r o\left(\underline{a}^{\prime}\right)}$ and $h_{c o(\underline{a})}$ do not depend on $\underline{b}^{\prime}$. It follows from P15 that

$$
\begin{aligned}
\sum_{\underline{b}^{\prime}} f_{\underline{c}, \underline{a}^{\prime}, \underline{b}^{\prime}}^{\prime} f_{\underline{a}, \underline{b}^{\prime}, \underline{b}} & =h_{r o\left(\underline{a}^{\prime}\right)} h_{c o(\underline{a})} \sum_{\underline{b}^{\prime}} g_{\sigma(\underline{c}), \sigma\left(\underline{a}^{\prime}\right), \sigma\left(\underline{b}^{\prime}\right)}^{\prime} g_{\sigma(\underline{a}), \sigma\left(\underline{b}^{\prime}\right), \sigma(\underline{b})} \\
& =h_{r o\left(\underline{a^{\prime}}\right)} h_{c o(\underline{a})} \sum_{\underline{b}^{\prime}} g_{\sigma(\underline{a}), \sigma(\underline{c}), \sigma\left(\underline{b}^{\prime}\right)} f_{\sigma\left(\underline{b^{\prime}}\right), \sigma\left(\underline{a}^{\prime}\right), \sigma(\underline{b})}^{\prime} \\
& =\sum_{\underline{b}^{\prime}} f_{\underline{a}, \underline{c}, \underline{b^{\prime}}} f_{\underline{b}^{\prime}, \underline{a}^{\prime}, \underline{b}}^{\prime} .
\end{aligned}
$$

Proposition 3.8. If $\underline{a} \sim_{L} \underline{b}$ and $\underline{a} \sim_{R} \underline{b}$, then $\underline{a}=\underline{b}$.

Proof. By [7, 4.2(a)-(e)] and [7, 4.4], the intersection of a left cell and a right cell both inside a two-sided cell contains exactly one element, as required.

\section{IRREDUCiBle CELl MOdules AND DUAL BASIS}

In this section we view the extension of scalars $K \mathcal{S}_{q}(n, r)$ of the $q$-Schur algebra $\mathcal{S}_{q}(n, r)$ as a symmetric algebra in the sense of [12, 7.1.1]. This is possible, since it is semisimple (see [1, (9.8)]). We can take as symmetrising trace form any $K$-linear form $\tau: K \mathcal{S}_{q}(n, r) \rightarrow K$ that is a $K$-linear combination

$$
\tau=\sum_{\chi \in \operatorname{Irr}\left(K \mathcal{S}_{q}(n, r)\right)} \frac{\chi}{c_{\chi}}
$$

of the irreducible characters where the $c_{\chi} \in K$ are non-zero constants, the so-called Schur elements (see [12, 7.1.1 and 7.2.6]). Clearly, $\tau$ is non-degenerate. Note that in [12, 7.2] it is shown that a symmetrising trace form uniquely determines the Schur elements via equation (4.1) and vice versa. Therefore, we will talk about Schur elements whenever a symmetrising trace form is given in the rest of this paper.

Having fixed $\tau$, we denote for any $K$-basis $\left(B_{\underline{a}}\right)_{\underline{a} \in M(n, r)}$ of $K \mathcal{S}_{q}(n, r)$ its dual basis with respect to $\tau$ by $\left(B_{\underline{b}}^{\vee}\right)_{\underline{b} \in M(n, r)}$. That is, we have $\tau\left(B_{\underline{a}} \cdot B_{\underline{b}}^{\vee}\right)=\tau\left(B_{\underline{b}}^{\vee} \cdot B_{\underline{a}}\right)=$ $\delta_{\underline{a}, \underline{b}}$ for all $\underline{a}, \underline{b} \in M(n, r)$. Note that this immediately implies that we can write every element $x \in K \mathcal{S}_{q}(n, r)$ in the following form:

$$
x=\sum_{\underline{a} \in M(n, r)} \tau\left(x \cdot B_{\underline{a}}^{\vee}\right) B_{\underline{a}}=\sum_{\underline{a} \in M(n, r)} \tau\left(x \cdot B_{\underline{a}}\right) B_{\underline{a}}^{\vee}
$$


(just write $x$ as a linear combination of the $B_{\underline{a}}$, multiply by some $B_{\underline{b}}$ and apply $\tau$ ).

Remark 4.1. We have $f_{\underline{a}, \underline{b}, \underline{c}}=\tau\left(\theta_{\underline{a}} \cdot \theta_{\underline{b}} \cdot \theta_{\underline{c}}^{\vee}\right)$ for all $\underline{a}, \underline{b}, \underline{c} \in M(n, r)$. Moreover, we note that formula (4.2) immediately gives us nice formulas for the matrix representations coming from the left cell modules. For a left cell $\Gamma$ and an element $h \in \mathcal{S}_{q}(n, r)$ the representing matrix of $h$ on the left cell module $\mathrm{LC}^{(\Gamma)}$ with respect to the basis $\left\{\theta_{\underline{a}}+\mathcal{S}_{<\Gamma} \mid \underline{a} \in \Gamma\right\}$ is $\left(\tau\left(\theta_{\underline{b}}^{\vee} \cdot h \cdot \theta_{\underline{a}}\right)\right)_{\underline{b}, \underline{a} \in \Gamma}$ since $h \cdot \theta_{\underline{a}}=\sum_{\underline{b} \in M(n, r)} \tau\left(\theta_{\underline{b}}^{\vee} \cdot h \cdot \theta_{\underline{a}}\right) \cdot \theta_{\underline{b}}$ and it is enough to sum over those $\underline{b}$ with $\underline{b} \leq_{L} \underline{a}$.

Lemma 4.2 (Characterisation of $\leq_{L}$ and $\leq_{R}$ ). We have $\underline{a} \leq_{L} \underline{b}$ if and only if $\theta_{\underline{b}} \theta_{\underline{a}}^{\vee} \neq 0$ and $\underline{a} \leq_{R} \underline{b}$ if and only if $\theta_{\underline{a}}^{\vee} \theta_{\underline{b}} \neq 0$.

Proof. We only show the version with $\leq_{L}$, the other is completely analogous thanks to Lemma 3.4. If $\underline{a} \leq_{L} \underline{b}$ there exists a $\underline{c} \in M(n, r)$ with $f_{\underline{c}, \underline{b}, \underline{a}}=\tau\left(\theta_{\underline{c}} \theta_{\underline{b}} \theta_{\underline{a}}^{\vee}\right) \neq 0$ which implies $\theta_{\underline{b}} \theta_{a}^{\vee} \neq 0$. If we assume the latter, then by the non-degeneracy of $\tau$ there is some $\underline{c} \in M(n, r)$ with $\tau\left(\theta_{\underline{c}} \theta_{\underline{b}} \theta_{\underline{a}}^{\vee}\right) \neq 0$ and $\underline{a} \leq_{L} \underline{b}$ follows.

The other major ingredient is the fact that cell modules are simple, more precisely:

Theorem 4.3 (Simple cell modules, see [6] or [7, 4.3]). Let $\Gamma$ be a left cell and recall $K=\mathbb{Q}(v)$. The extension of scalars $K \mathrm{LC}^{(\Gamma)}$ of the left cell module $\mathrm{LC}^{(\Gamma)}$ for a left cell $\Gamma$ is a simple $K \mathcal{S}_{q}(n, r)$-module.

Proof. See [6] or [7, 4.3].

Remark 4.4. This, in particular, implies that all simple $K \mathcal{S}_{q}(n, r)$-modules can be realised over the ring $A$, since their corresponding representing matrices involve only structure constants of $\mathcal{S}_{q}(n, r)$.

We now directly obtain useful algebra elements by using the simple cell modules:

Theorem 4.5 (Basis of an isotypic component). Let $\Gamma$ be a left cell and $\chi$ the corresponding irreducible character of the left cell module $\mathrm{LC}^{(\Gamma)}$, then the elements

$$
\left(c_{\chi}^{-1} \theta_{\underline{a}} \theta_{\underline{b}}^{\vee}\right)_{\underline{a}, \underline{b} \in \Gamma}
$$

are $K$-linearly independent and span the isotypic component of $K \mathcal{S}_{q}(n, r)$ belonging to the character $\chi$. Furthermore, we have the relations

$$
\left(c_{\chi}^{-1} \theta_{\underline{a}} \theta_{\underline{b}}^{\vee}\right) \cdot\left(c_{\chi}^{-1} \theta_{\underline{a^{\prime}}} \theta_{\underline{b^{\prime}}}^{\vee}\right)=\delta_{\underline{b}, \underline{a}^{\prime}} \cdot c_{\chi}^{-1} \theta_{\underline{a}} \theta_{\underline{b}^{\prime}}^{\vee}
$$

for all $\underline{a}, \underline{b}, \underline{a}^{\prime}, \underline{b}^{\prime} \in \Gamma$. That is, these elements form a matrix unit for the isotypic component of $K \mathcal{S}_{q}(n, r)$ corresponding to the simple module $K \mathrm{LC}^{(\Gamma)}$.

Proof. By [12, 7.2.7] we get a matrix unit for the isotypic component of $K \mathcal{S}_{q}(n, r)$ corresponding to the simple module $K \mathrm{LC}^{(\Gamma)}$ by the elements

$$
\frac{1}{c_{\chi}} \sum_{\underline{c} \in M(n, r)} \tau\left(\theta_{\underline{b}}^{\vee} \cdot \theta_{\underline{c}} \cdot \theta_{\underline{a}}\right) \cdot \theta_{\underline{c}}^{\vee}=\frac{1}{c_{\chi}} \sum_{\underline{c} \in M(n, r)} \tau\left(\theta_{\underline{c}} \cdot \theta_{\underline{a}} \theta_{\underline{b}}^{\vee}\right) \cdot \theta_{\underline{c}}^{\vee}
$$

for $\underline{a}, \underline{b} \in \Gamma$. But this is equal to $c_{\chi}^{-1} \theta_{\underline{a}} \theta_{\underline{b}}^{\vee}$ by formula (4.2). 
Corollary 4.6. Let $\Gamma$ be a left cell and $\chi$ the corresponding irreducible character of the left cell module $\mathrm{LC}^{(\Gamma)}$. Then the element

$$
e_{\Gamma}:=\frac{1}{c_{\chi}} \sum_{\underline{a} \in \Gamma} \theta_{\underline{a}} \theta_{\underline{a}}^{\vee}
$$

is the central primitive idempotent of $K \mathcal{S}_{q}(n, r)$ corresponding to the irreducible character $\chi$.

Proof. By Theorem $4.5 e_{\Gamma}$ lies in the isotypic component corresponding to the character $\chi$ and is mapped to the identity matrix in the corresponding matrix representation.

Lemma 4.7 (Isomorphism of left cell modules and two-sided cells). Let $\Gamma$ and $\Gamma^{\prime}$ be left cells. If $K \mathrm{LC}^{(\Gamma)}$ and $K \mathrm{LC}^{\left(\Gamma^{\prime}\right)}$ are isomorphic $K \mathcal{S}_{q}(n, r)$-modules, then $\Gamma$ and $\Gamma^{\prime}$ lie in the same two-sided cell.

Proof. Let $\chi$ be the irreducible character of the left cell module $\mathrm{LC}^{(\Gamma)}$ and $\chi^{\prime}$ that of $\mathrm{LC}^{\left(\Gamma^{\prime}\right)}$. The modules $K \mathrm{LC}^{(\Gamma)}$ and $K \mathrm{LC}^{\left(\Gamma^{\prime}\right)}$ are isomorphic if and only if $e_{\Gamma} \cdot e_{\Gamma^{\prime}}=e_{\Gamma^{\prime}} \cdot e_{\Gamma} \neq 0$ (and in this case $e_{\Gamma}=e_{\Gamma^{\prime}}$ ). Now assume this case. Then

$$
0 \neq \frac{1}{c_{\chi}^{2}} \sum_{\underline{a} \in \Gamma \underline{\underline{b} \in \Gamma^{\prime}}} \theta_{\underline{a}} \theta_{\underline{a}}^{\vee} \theta_{\underline{b}} \theta_{\underline{b}}^{\vee}=\frac{1}{c_{\chi}^{2}} \sum_{\underline{a} \in \Gamma} \sum_{\underline{b} \in \Gamma^{\prime}} \theta_{\underline{b}} \theta_{\underline{b}}^{\vee} \theta_{\underline{a}} \theta_{\underline{a}}^{\vee}
$$

and thus there is at least one pair $(\underline{a}, \underline{b}) \in \Gamma \times \Gamma^{\prime}$ such that $\theta_{a}^{\vee} \theta_{\underline{b}} \neq 0$. By Lemma 4.2 this implies $\underline{a} \leq_{R} \underline{b}$. Since $e_{\Gamma}$ and $e_{\Gamma^{\prime}}$ commute, the same argument shows $\underline{b}^{\prime} \leq_{R} \underline{a}^{\prime}$ for some $\underline{a}^{\prime} \in \Gamma$ and $\underline{b}^{\prime} \in \Gamma^{\prime}$. Thus, $\Gamma$ and $\Gamma^{\prime}$ lie in the same two-sided cell in that case.

For what follows we need the following statement about Iwahori-Hecke algebras of type A:

Theorem 4.8 (Equal cell modules in the Iwahori-Hecke algebra). Let $\mathcal{H}$ be a generic Iwahori-Hecke algebra of type $A$ as in Section 2. Let $\tau_{0}$ be the standard symmetrising trace form on $\mathcal{H}$,

$$
\tau_{0}\left(\sum_{w \in W} a_{w} T_{w}\right):=a_{1},
$$

where $1 \in W$ denotes the identity. We denote by $\left\{D_{w^{-1}}\right\}_{w \in W}$ the dual basis of $\left\{C_{w}\right\}_{w \in W}$ with respect to $\tau_{0}$, that is, we have $\tau_{0}\left(C_{w} \cdot D_{x^{-1}}\right)=\delta_{w, x}$ for $w, x \in W$.

If $x \sim_{L} y$ and $z \sim_{L} w$ and $x \sim_{R} z$ and $y \sim_{R} w$, then $C_{x} D_{y^{-1}}=C_{z} D_{w^{-1}}$. In particular, we have

$$
g_{u, x, y}=\tau_{0}\left(C_{u} C_{x} D_{y^{-1}}\right)=\tau_{0}\left(C_{u} C_{z} D_{w^{-1}}\right)=g_{u, z, w}
$$

for all $u \in W$.

Proof. This statement is already implicitly stated in [14. Namely, it is shown there in the proof of Theorem 1.4 that the two left cell modules defined by the left cell containing $x, y$ and the one containing $z, w$ are isomorphic since all four lie in the same two-sided cell. The exact statement there is that two $W$-graphs are isomorphic, which means, in particular, that not only the two left cell modules 
are isomorphic, but that even the matrix representations with respect to the bases $\left\{C_{v} \mid v \sim_{L} x\right\}$ and $\left\{C_{w} \mid w \sim_{L} z\right\}$ are equal. But this exactly means, that

$$
\tau_{0}\left(C_{u} C_{x} D_{y^{-1}}\right)=\tau_{0}\left(D_{y^{-1}} C_{u} C_{x}\right)=\tau_{0}\left(D_{w^{-1}} C_{u} C_{z}\right)=\tau_{0}\left(C_{u} C_{z} D_{w^{-1}}\right)
$$

for all $u \in W$ which we claim. For more details see [17, 1.7.3].

Remark 4.9. Note that Theorem 4.8 implies that the bases for a Specht module labelled by a two-sided cell constructed in [9, Lemma 6.1] are all the same.

Now we begin to use statements Q1 to Q14:

Theorem 4.10 (Equality of different left cell modules). Let $\Gamma, \Gamma^{\prime}$ be left cells such that $K \mathrm{LC}^{(\Gamma)}$ and $K \mathrm{LC}^{\left(\Gamma^{\prime}\right)}$ are isomorphic $K \mathcal{S}_{q}(n, r)$-modules. Let $\underline{d}$ be the unique element in $\Gamma^{\prime} \cap \mathcal{D}(n, r)$ (use Q13) and $\underline{c} \sim_{L} \underline{d}$ that is $\underline{c} \in \Gamma^{\prime}$. Then there are unique $\underline{a}, \underline{b} \in \Gamma$ with $\underline{a} \sim_{R} \underline{c}$ and $\underline{b} \sim_{R} \underline{d}$ and we have $\theta_{\underline{a}} \theta_{\underline{b}}^{\vee}=\theta_{\underline{c}} \theta_{\underline{d}}^{\vee}$.

Proof. Let $\chi$ be the irreducible character of the left cell module $\mathrm{LC}^{\Gamma^{\prime}}$. We denote by $c_{\chi}$ the corresponding Schur element. Since $\underline{c} \sim_{L} \underline{d}$, it follows from Theorem 4.5 that

$$
\theta_{\underline{d}} \theta_{\underline{c}}^{\vee} \theta_{\underline{c}} \theta_{\underline{d}}^{\vee}=c_{\chi} \theta_{\underline{d}} \theta_{\underline{d}}^{\vee}
$$

Therefore, we have $\tau\left(\theta_{\underline{c}}^{\vee} \theta_{\underline{c}} \theta_{\underline{d}}^{\vee} \theta_{\underline{d}}\right) \neq 0$ and hence $\theta_{\underline{c}} \theta_{\underline{d}}^{\vee}$ acts non-trivially on the module $\mathrm{LC}^{\left(\Gamma^{\prime}\right)}$ (see Remark 4.1) and thus also on the isomorphic module $\mathrm{LC}^{(\Gamma)}$.

This means that there is at least one pair $(\underline{a}, \underline{b}) \in \Gamma \times \Gamma$ such that

$$
\tau\left(\theta_{\underline{b}} \theta_{\underline{a}}^{\vee} \cdot \theta_{\underline{c}} \theta_{\underline{d}}^{\vee}\right)=\tau\left(\theta_{\underline{a}}^{\vee} \cdot \theta_{\underline{c}} \theta_{\underline{d}}^{\vee} \cdot \theta_{\underline{b}}\right)=\tau\left(\theta_{\underline{c}} \theta_{\underline{d}}^{\vee} \cdot \theta_{\underline{b}} \theta_{\underline{a}}^{\vee}\right) \neq 0 .
$$

But then, in particular, $\theta_{\underline{a}}^{\vee} \theta_{\underline{c}} \neq 0$ and thus $a \leq_{R} c$ by Lemma 4.2. Since $\Gamma$ and $\Gamma^{\prime}$ lie in the same two-sided cell by Lemma 4.7. we conclude $\underline{a} \sim_{L R} \underline{c}$ and thus by Q4 and Q10 $\underline{a} \sim_{R} \underline{c}$. Analogously, we show $\underline{b} \sim_{R} \underline{d}$. By Proposition 3.8 we conclude that there is only one such pair $(\underline{a}, \underline{b})$ since both are uniquely defined by their membership in a left and a right cell.

We now show that $f_{\underline{e}, \underline{a}, \underline{b}}=f_{\underline{e}, \underline{c}, \underline{d}}$ for all $\underline{e} \in M(n, r)$ and thus $\theta_{\underline{a}} \theta_{\underline{b}}^{\vee}=\theta_{\underline{c}} \theta_{\underline{d}}^{\vee}$. We have $c o(\underline{a})=c o(\underline{b})$ and $c o(\underline{c})=c o(\underline{d})=\operatorname{ro}(\underline{d})=\operatorname{ro}(\underline{b})$ and $r o(\underline{a}) \stackrel{\underline{a}}{=} \operatorname{ro}(\underline{c})$ by Lemma $\underline{3.2}$ and the fact that $\underline{d} \in \mathcal{D}(n, r)$. Thus, if $r o(\underline{e}) \neq r o(\underline{b})$ or $c o(\underline{e}) \neq \operatorname{ro}(\underline{a})$, then both sides are zero by Lemma 2.3. Otherwise, we have

$$
f_{\underline{e}, \underline{a}, \underline{b}}=h_{c o(\underline{e})}^{-1} \cdot g_{\sigma(\underline{e}), \sigma(\underline{a}), \sigma(\underline{b})} \quad \text { and } \quad f_{\underline{e}, \underline{c}, \underline{d}}=h_{c o(\underline{e})}^{-1} \cdot g_{\sigma(\underline{e}), \sigma(\underline{c}), \sigma(\underline{d})}
$$

and thus the equality $f_{\underline{e}, \underline{a}, \underline{b}}=f_{\underline{e}, \underline{c}, \underline{d}}$ follows from

$$
\sigma(\underline{a}) \sim_{L} \sigma(\underline{b}) \sim_{R} \sigma(\underline{d}) \sim_{L} \sigma(\underline{c}) \sim_{R} \sigma(\underline{a})
$$

using Lemma 3.1 and Theorem 4.8. The non-degeneracy of $\tau$ now immediately implies $\theta_{\underline{a}} \theta_{\underline{b}}^{\vee}=\theta_{\underline{c}} \theta_{\underline{d}}^{\vee}$.

With this we get the following result, for which we first need one more piece of notation:

Definition 4.11 (Schur elements of characters of left cell modules). Let $\underline{d} \in \mathcal{D}(n, r)$ and $\Gamma$ the unique left cell with $\underline{d} \in \Gamma$ (remember Q13). We denote the left cell module $\mathrm{LC}^{(\Gamma)}$ by $\mathrm{LC}^{(\underline{d})}$ and the Schur element corresponding to the irreducible character of $\mathrm{LC}^{(\underline{d})}$ by $c_{\underline{d}}$. Note that $c_{\underline{d}}=c_{\underline{d}^{\prime}}$ if the two left cell modules LC $\mathrm{C}^{(\underline{d})}$ and $\mathrm{LC}^{\left(\underline{d}^{\prime}\right)}$ are isomorphic, because by $[7,4.3 .1,4.4 .1], d$ and $d^{\prime}$ lie in the same two-sided cell if and only if $\mathrm{LC}^{(\underline{d})}$ is isomorphic to $\mathrm{LC}^{\left(\underline{d}^{\prime}\right)}$. 
Theorem 4.12 (Wedderburn basis). Let $\tau$ be an arbitrary symmetrising trace form (that is, non-degenerate) on $K \mathcal{S}_{q}(n, r)$. The set

$$
\mathcal{B}:=\left\{c_{\underline{d}}^{-1} \theta_{\underline{c}} \theta_{\underline{d}}^{\vee} \mid \underline{c} \in M(n, r), \underline{d} \in \mathcal{D}(n, r), \underline{c} \sim_{L} \underline{d}\right\}
$$

is a Wedderburn basis of $K \mathcal{S}_{q}(n, r)$. Two elements $c_{\underline{d}}^{-1} \theta_{\underline{c}} \theta_{\underline{d}}^{\vee}$ and $c_{\underline{d^{\prime}}}^{-1} \theta_{{\underline{c^{\prime}}}^{\prime}} \theta_{\underline{d}^{\prime}}^{\vee}$ lie in the same isotypic component if and only if $\mathrm{LC}^{(\underline{d})} \cong \mathrm{LC}^{\left(\underline{d}^{\prime}\right)}$.

For $c_{\underline{d}}^{-1} \theta_{\underline{c}} \theta_{\underline{d}}^{\vee}, c_{\underline{d^{\prime}}}^{-1} \theta_{{\underline{c^{\prime}}}^{\prime}} \theta_{\underline{d^{\prime}}}^{\vee} \in \mathcal{B}$ we have the following equation:

$$
c_{\underline{d}}^{-1} \theta_{\underline{c}} \theta_{\underline{d}}^{\vee} \cdot c_{\underline{d^{\prime}}}^{-1} \theta_{{\underline{c^{\prime}}}^{\prime}} \theta^{\vee}= \begin{cases}0 & \text { if } \mathrm{LC}^{(\underline{d})} \approx \mathrm{LC}^{\left(\underline{d^{\prime}}\right)}, \\ 0 & \text { if } \mathrm{LC}^{(\underline{d})} \cong \mathrm{LC}^{\left(\underline{d^{\prime}}\right)} \text { and } \underline{d} \psi_{R} \underline{c^{\prime}}, \\ c_{\underline{d^{\prime}}}^{-1} \theta_{\underline{c^{\prime \prime}}} \theta_{\underline{d^{\prime}}}^{\vee} & \text { if } \mathrm{LC}^{(\underline{d})} \cong \mathrm{LC}^{\left(\underline{d^{\prime}}\right)} \text { and } \underline{d} \sim_{R} \underline{c^{\prime}} .\end{cases}
$$

Here, $\underline{c^{\prime \prime}}$ in the last case is the unique element with $\underline{c^{\prime \prime}} \sim_{L} \underline{d^{\prime}}$ and $\underline{c^{\prime \prime}} \sim_{R} \underline{c}$ and the statement contains the information that such a $\underline{c}^{\prime \prime}$ in fact exists.

Proof. By Theorem 4.5 the elements $c_{\underline{d}}^{-1} \theta_{\underline{c}} \theta_{\underline{d}}^{\vee}$ and $c_{\underline{d^{\prime}}}^{-1} \theta_{{\underline{c^{\prime}}}^{\prime}} \theta_{\underline{d^{\prime}}}^{\vee}$ both lie in an isotypic component. Thus, if $\mathrm{LC}^{(\underline{d})} \neq \mathrm{LC}^{\left(\underline{d}^{\prime}\right)}$, then clearly their product is zero.

Now assume that the left cell modules are isomorphic. Let $\Gamma$ be an arbitrary left cell, such that $K \mathrm{LC}^{(\Gamma)}$ is isomorphic to $K \mathrm{LC}^{(\underline{d})}$ and $K \mathrm{LC}^{\left(\underline{d}^{\prime}\right)}$ and denote the corresponding irreducible character by $\chi$. By Theorem 4.10 there are unique $\underline{a}, \underline{b}, \underline{a^{\prime}}, \underline{b^{\prime}} \in \Gamma$ with

$$
\underline{a} \sim_{R} \underline{c} \text { and } \underline{b} \sim_{R} \underline{d} \quad \text { and } \quad \underline{a^{\prime}} \sim_{R} \underline{c^{\prime}} \quad \text { and } \quad \underline{b^{\prime}} \sim_{R} \underline{d^{\prime}}
$$

and we have $\theta_{\underline{a}} \theta_{\underline{b}}^{\vee}=\theta_{\underline{c}} \theta_{\underline{d}}^{\vee}$ and $\theta_{\underline{a^{\prime}}} \theta_{\underline{b}^{\prime}}^{\vee}=\theta_{\underline{c}^{\prime}} \theta_{\underline{d}^{\prime}}^{\vee}$. Thus, Theorem 4.5] implies that the product in the theorem is 0 if $\underline{b} \not=\underline{a^{\prime}}$ and equal to $c_{\chi}^{-1} \theta_{\underline{a}} \theta_{\underline{b}^{\prime}}^{\vee}$ otherwise. We remark that if $\underline{d} \sim_{R} \underline{c}^{\prime}$, then $\underline{a^{\prime}} \sim_{R} \underline{b}$ by transitivity. But using Proposition $3.8, \underline{a^{\prime}}, \underline{b} \in \Gamma$ implies $\underline{b}=\underline{a^{\prime}}$. Hence $\underline{b}=\underline{a^{\prime}}$ if and only if $\underline{d} \sim_{R} \underline{c}^{\prime}$ which proves case two in the equation.

Finally, we assume also that $\underline{d} \sim_{R} \underline{c^{\prime}}$. Then, as $\underline{c^{\prime \prime}}$ runs through the left cell that contains $\underline{d^{\prime}}$, we can apply Theorem 4.10 to each $\theta_{{\underline{c^{\prime \prime}}}^{\prime}} \theta_{\underline{d}^{\prime}}^{\vee}$ and the left cell $\Gamma$. Since $\underline{b^{\prime}} \in \Gamma$ and $\underline{b^{\prime}} \sim_{R} \underline{d^{\prime}}$ we get that

$$
\left\{\theta_{\underline{c^{\prime \prime}}} \theta_{\underline{d^{\prime}}}^{\vee} \mid \underline{c^{\prime \prime}} \sim_{L} \underline{d^{\prime}}\right\}=\left\{\theta_{\underline{a^{\prime \prime}}} \theta_{\underline{b^{\prime}}}^{\vee} \mid \underline{a^{\prime \prime}} \in \Gamma\right\}
$$

and both sets have cardinality $|\Gamma|$. Thus, there is a unique $\underline{c^{\prime \prime}}$ with $\theta_{\underline{c^{\prime \prime}}} \theta_{\underline{d^{\prime}}}^{\vee}=\theta_{\underline{a}} \theta_{\underline{b^{\prime}}}^{\vee}$ characterised by $\underline{a} \sim_{R} \underline{c^{\prime \prime}} \sim_{L} \underline{d^{\prime}}$ and the theorem is proved.

Corollary 4.13 (Idempotents). The elements $c_{\underline{d}}^{-1} \theta_{\underline{d}} \theta_{\underline{d}}^{\vee}$ with $\underline{d} \in \mathcal{D}(n, r)$ are pairwise orthogonal primitive idempotents whose sum is the identity $1 \in \mathcal{S}_{q}(n, r)$. The central primitive idempotent corresponding to an irreducible character $\chi$ of $K \mathcal{S}_{q}(n, r)$ is equal to

$$
\sum_{\mathrm{LC}^{(\underline{d})} \text { has character } \chi} c_{\underline{d}}^{-1} \theta_{\underline{d}} \theta_{\underline{d}}^{\vee} .
$$

Proof. This follows directly from Theorems 4.12, 4.10 and 4.5

Corollary 4.14 (Left cell modules as submodules). Let $\underline{d} \in \mathcal{D}(n, r)$. Then the A-span

$$
\mathcal{L}_{\underline{d}}:=\left\langle\theta_{\underline{c}} \theta_{\underline{d}}^{\vee} \mid \underline{c} \sim_{L} \underline{d}\right\rangle_{A}
$$

is a left $\mathcal{S}_{q}(n, r)$-module by the multiplication in $K_{\mathcal{S}}(n, r)$ that is isomorphic to the left cell module $\mathrm{LC}^{(\underline{d})}$. In fact, the representing matrices with respect to the basis 
$\left(\theta_{\underline{c}} \theta_{d}^{\vee}\right)_{\underline{\underline{c}} \sim_{L} \underline{d}}$ are equal to the representing matrices coming from the left cell module $\mathrm{LC}^{(\bar{d})}$ with respect to its standard basis.

Proof. Let $\Gamma$ be the left cell that contains $\underline{d}$. Then by Formula (4.2) we have for every $h \in \mathcal{S}_{q}(n, r)$ :

$$
h \theta_{\underline{c}}=\sum_{\underline{c^{\prime}} \in M(n, r)} \tau\left(\theta_{\underline{c^{\prime}}}^{\vee} \cdot h \theta_{\underline{c}}\right) \cdot \theta_{\underline{c^{\prime}}} .
$$

Moreover, for $\underline{a} \in A$, there is $\alpha_{\underline{a}} \in A$ such that

$$
h=\sum_{\underline{a} \in M(n, r)} \alpha_{\underline{a}} \theta_{\underline{a}} .
$$

Hence, for $\underline{c}, \underline{c^{\prime}} \in M(n, r)$, we have $\tau\left(\theta_{\underline{c^{\prime}}}^{\vee} \cdot h \theta_{\underline{c}}\right) \in A$, because $\tau\left(\theta_{\underline{c}^{\prime}}^{\vee} \cdot \theta_{\underline{a}} \theta_{\underline{c}}\right) \in A$ (see Remark 4.1). Multiplying this from the right with $\theta_{\underline{d}}^{\vee}$ we get

$$
h \theta_{\underline{c}} \theta_{\underline{d}}^{\vee}=\sum_{\underline{c^{\prime}} \in M(n, r)} \tau\left(h \theta_{\underline{c}} \theta_{\underline{c^{\prime}}}^{\vee}\right) \cdot \theta_{\underline{c^{\prime}}} \theta_{\underline{d}}^{\vee},
$$

where we only have to sum over $\underline{c}^{\prime} \in \Gamma$, since all the summands are zero unless $\underline{d} \leq_{L} \underline{c^{\prime}} \leq_{L} \underline{c}$ by Lemma 4.2, which is equivalent to $\underline{c^{\prime}} \in \Gamma$. We then deduce that $\mathcal{L}_{\underline{d}}$ is a left $\mathcal{S}_{q}(n, r)$-module. Moreover, comparing with Remark 4.1, this shows the statement of the representing matrices.

Corollary 4.15. The Schur algebra $\mathcal{S}_{q}(n, r)$ is contained in the A-span of the Wedderburn basis $\mathcal{B}$ :

$$
\mathcal{S}_{q}(n, r) \subseteq\langle\mathcal{B}\rangle_{A}
$$

Proof. Let $\Gamma_{1}, \ldots, \Gamma_{n}$ be left cells, such that the corresponding left cell modules form a system of representatives for the isomorphism types of simple left $K \mathcal{S}_{q}(n, r)$ modules. The mapping that maps $h \in K \mathcal{S}_{q}(n, r)$ to its tuple of representing matrices in the cell modules $\mathrm{LC}^{\left(\Gamma_{1}\right)}, \ldots, \mathrm{LC}^{\left(\Gamma_{n}\right)}$ with respect to their standard basis is an explicit isomorphism to a direct sum of full matrix rings over $K$. In this isomorphism, the elements of $\mathcal{B}$ are mapped to a matrix unit, that is, to tuples of matrices, in which exactly one matrix is non-zero, and this matrix contains exactly one non-zero coefficient equal to 1 . The elements of $\mathcal{S}_{q}(n, r)$ are mapped to tuples of matrices with entries in $A$, since their representing matrices on the cell modules have entries in $A$ (see the remark after Theorem 4.3). Therefore, $\mathcal{S}_{q}(n, r)$ lies in the $A$-span of $\mathcal{B}$.

Proposition 4.16. Let $\tau$ be a symmetrising trace form on $K \mathcal{S}_{q}(n, r)$. We denote by $\mathcal{B}$ the corresponding Wedderburn basis obtained in Theorem 4.12 . Then, the dual basis of $\mathcal{B}$ relative to $\tau$ is

$$
\mathcal{B}^{\vee}=\left\{\theta_{\underline{c}} \theta_{\underline{d}}^{\vee} \mid \underline{c} \in M(n, r), \underline{d} \in \mathcal{D}(n, r), \underline{c} \sim_{L} \underline{d}\right\} .
$$

Proof. Note first, that since $\tau$ is non-degenerate and $\mathcal{B}$ is a basis of $K \mathcal{S}_{q}(n, r)$, there must be at least one element $c_{\underline{d^{\prime}}}^{-1} \theta_{\underline{c^{\prime}}} \theta_{\underline{d^{\prime}}}^{\vee} \in \mathcal{B}$ such that $\tau\left(c_{\underline{d}}^{-1} \theta_{\underline{c}} \theta_{\underline{d}}^{\vee} \cdot c_{\underline{d}^{\prime}}^{-1} \theta_{\underline{c^{\prime}}} \theta_{\underline{d^{\prime}}}^{\vee}\right)$ is non-zero. Since $c_{\underline{d}^{\prime}} \neq 0$, we have, in particular, $\tau\left(c_{\underline{d}}^{-1} \theta_{\underline{c}} \theta_{\underline{d}}^{\vee} \theta_{{\underline{c^{\prime}}}^{\prime}} \theta_{\underline{d}^{\prime}}^{\vee}\right) \neq 0$. We try to find out, which element $\theta_{{\underline{c^{\prime}}}^{\prime}} \theta_{{\underline{d^{\prime}}}^{\prime}}^{\vee}$ this can be:

By Theorem 4.12, the value $\tau\left(c_{d}^{-1} \theta_{\underline{c}} \theta_{\underline{d}}^{\vee} \theta_{\underline{c^{\prime}}} \theta_{\underline{d^{\prime}}}^{\vee}\right)$ is equal to zero, if $\mathrm{LC}^{(\underline{d})} \not \mathrm{LC}^{\left(d^{\prime}\right)}$ or $\underline{d} \chi_{R} \underline{c^{\prime}}$. If, however, $\mathrm{LC}^{(\underline{d})} \cong \mathrm{LC}^{\left(d^{\prime}\right)}$ and $\underline{d} \sim_{R} \underline{c^{\prime}}$, then it is equal to $\tau\left(\theta_{\underline{c}^{\prime \prime}} \theta_{\underline{d}^{\prime}}\right)$ where $\underline{c^{\prime \prime}}$ is uniquely defined by $\underline{c^{\prime \prime}} \sim_{L} \underline{d^{\prime}}$ and $\underline{c^{\prime \prime}} \sim_{R} \underline{c}$. If $\underline{c^{\prime \prime}} \neq \underline{d^{\prime}}$, then this value 
is also equal to 0 because of the original definition of $\left\{\theta_{a}^{\vee} \mid \underline{a} \in M(n, r)\right\}$. If, however, $\underline{c^{\prime \prime}}=\underline{d^{\prime}}$, we can show that $\underline{c}^{\prime}=\underline{c}^{t}$ using Proposition 3.8. Namely, we have $\underline{c^{\prime}} \sim_{L} \underline{d^{\prime}}=\underline{c^{\prime \prime}} \sim_{R} \underline{c}$ and thus $\underline{c^{\prime}} \sim_{L} \underline{c}^{t}$ by transposition. Further, we have $\underline{c^{\prime}} \sim_{R} \underline{d} \sim_{L} \underline{c}$ and thus again by transposition $\underline{c}^{\prime} \sim_{R} \underline{c}^{t}$. Thus, $\underline{c}^{\prime}$ and $\underline{c}^{t}$ are both left and right equivalent and therefore equal.

Thus, we deduce that

$$
\tau\left(c_{\underline{d}}^{-1} \theta_{\underline{c}} \theta_{\underline{d}}^{\vee} \cdot \theta_{\underline{c^{\prime}}} \theta_{\underline{d^{\prime}}}^{\vee}\right)=\delta_{\underline{c^{\prime}}, \underline{c^{t}}}
$$

for all $\underline{c} \in M(n, r)$ and $\underline{d} \in \mathcal{D}(n, r)$ with $\underline{c} \sim_{L} \underline{d}$, and all $\underline{c}^{\prime} \in M(n, r)$ and $\underline{d^{\prime}} \in \mathcal{D}(n, r)$ with $\underline{c^{\prime}} \sim_{L} \underline{d^{\prime}}$.

Remark 4.17. Note that as a byproduct we have proved the following result: If $\underline{c} \in M(n, r)$ and $\underline{d} \in \mathcal{D}(n, r)$ with $\underline{c} \sim_{L} \underline{d}$, and $\underline{d^{\prime}} \in \mathcal{D}(n, r)$ with $\underline{c}^{t} \sim_{L} \underline{d^{\prime}}$, then $\mathrm{LC}^{(\underline{d})} \cong \mathrm{LC}^{\left(\underline{d^{\prime}}\right)}$.

We now talk about $A$-sublattices of $K \mathcal{S}_{q}(n, r)$.

Definition/Proposition 4.18 ( $A$-sublattices of $K \mathcal{S}_{q}(n, r)$ and their duals). By an $A$-lattice in $K \mathcal{S}_{q}(n, r)$ we mean an $A$-free $A$-submodule that contains a $K$-basis of $K \mathcal{S}_{q}(n, r)$. Let $L \subseteq K \mathcal{S}_{q}(n, r)$ be an $A$-lattice. Then we set

$$
L^{\vee}:=\left\{h \in K \mathcal{S}_{q}(n, r) \mid \tau(h x) \in A \text { for all } x \in L\right\}
$$

and call it the dual lattice of $L$. Since $\tau$ is non-degenerate, $L^{\vee}$ is again an $A$ lattice in $K \mathcal{S}_{q}(n, r)$, namely, if $\left(b_{\underline{a}}\right)_{a} \in M(n, r)$ is an $A$-basis of $L$, then the dual basis $\left(b_{\underline{a}}^{\vee}\right)_{a} \in M(n, r)$ is an $A$-basis of $L^{\vee}$. Clearly, if $L \subseteq N$ are two $A$-lattices in $K \mathcal{S}_{q}(n, r)$, then $N^{\vee} \subseteq L^{\vee}$.

Note that we do not require an $A$-lattice to be an $A$-algebra!

Proposition 4.19 (The dual is an $\mathcal{S}_{q}(n, r)$-module). We have $\mathcal{S}_{q}(n, r) \cdot \mathcal{S}_{q}(n, r)^{\vee} \subseteq$ $\mathcal{S}_{q}(n, r)^{\vee}$.

Proof. Fix $h \in \mathcal{S}_{q}(n, r)$ and $k \in \mathcal{S}_{q}(n, r)^{\vee}$. We have to show that $h k \in \mathcal{S}_{q}(n, r)^{\vee}$. However, for every $x \in \mathcal{S}_{q}(n, r)$ holds $\tau(h k x)=\tau(k x h)$. Since $x h \in \mathcal{S}_{q}(n, r)$ (because $\mathcal{S}_{q}(n, r)$ is an algebra), and $k \in \mathcal{S}_{q}(n, r)^{\vee}$ we get $\tau(k x h) \in A$.

For the rest of this section we let $\tau=\sum_{\chi \in \operatorname{Irr}\left(K \mathcal{S}_{q}(n, r)\right)} \chi$, that is, we choose $\tau$ such that all Schur elements are equal to 1.

Proposition 4.20 (The Wedderburn-basis is self-dual). Let $\tau=\sum_{\chi \in \operatorname{Irr}\left(K \mathcal{S}_{q}(n, r)\right)} \chi$. Then

$$
\langle\mathcal{B}\rangle_{A}^{\vee}=\langle\mathcal{B}\rangle_{A}
$$

for the Wedderburn basis $\mathcal{B}$ from Theorem 4.12 .

Proof. Since $\tau$ is the sum of the irreducible characters, all Schur elements $c_{\chi}$ are equal to one. It is then a direct consequence of Proposition 4.16 .

Corollary 4.21 (The dual of $\mathcal{S}_{q}(n, r)$ ). From Lemma 4.15 and Proposition 4.20 follows

$$
\langle\mathcal{B}\rangle_{A} \subseteq \mathcal{S}_{q}(n, r)^{\vee}
$$

Proof. Dualising reverses inclusion. 


\section{The Asymptotic Algebra And the Du-Lusztig homomorphism}

In this section we briefly recall the definition of the asymptotic algebra $\mathcal{J}(n, r)$ for the $q$-Schur algebra $\mathcal{S}_{q}(n, r)$ and of the Du-Lusztig homomorphism $\Phi$ from $\mathcal{S}_{q}(n, r)$ to $\mathcal{J}(n, r)$. We then show that this algebra is isomorphic to the algebra $\langle\mathcal{B}\rangle_{A}$ spanned by our Wedderburn basis $\mathcal{B}$ and that the Du-Lusztig homomorphism can be interpreted as the inclusion of $\mathcal{S}_{q}(n, r)$ into $\langle\mathcal{B}\rangle_{A}$.

Definition 5.1 (The asymptotic algebra $\mathcal{J}(n, r)$ ). Let $\mathcal{J}(n, r)$ be the free abelian group with basis $\left\{t_{\underline{a}} \mid \underline{a} \in M(n, r)\right\}$. We define a multiplication on $\mathcal{J}(n, r)$ by setting

$$
t_{\underline{a}} t_{\underline{b}}=\sum_{\underline{c} \in M(n, r)} \gamma_{\underline{a}, \underline{b}, \underline{c}^{t}} \cdot t_{\underline{c}}
$$

We set $\mathcal{D}(n, r)_{\lambda}:=\mathcal{D}(n, r) \cap M_{\lambda, \lambda}$. Following Du, we denote the extension of scalars of $\mathcal{J}(n, r)$ to $A$ by $\mathcal{J}(n, r)_{A}$.

Lemma 5.2 (See [7, (2.2.1)]). The $\mathbb{Z}$-algebra $\mathcal{J}(n, r)$ is associative with the identity element

$$
\sum_{\underline{d} \in \mathcal{D}(n, r)} t_{\underline{d}}
$$

Theorem 5.3 (The Du-Lusztig homomorphism $\Phi$, see [7, (2.3]). The A-linear map $\Phi: \mathcal{S}_{q}(n, r) \rightarrow \mathcal{J}(n, r)_{A}$ defined by

$$
\Phi\left(\theta_{\underline{a}}\right):=\sum_{\substack{b \in M(n, r) \\ \bar{d} \in \mathcal{D}(n, r)_{\mu} \\ \mathbf{a}(\underline{d})=\mathbf{a}(\underline{b})}} f_{\underline{a}, \underline{d}, \underline{b}} \cdot t_{\underline{b}}=\sum_{\substack{\underline{b} \in M(n, r) \\ \underline{d} \in \mathcal{D}(n, r) \\ \underline{d} \sim_{L} \underline{b}}} f_{\underline{a}, \underline{d}, \underline{b}} \cdot t_{\underline{b}}, \quad \text { where } \mu=\operatorname{co}(\underline{a})
$$

is an algebra homomorphism and becomes an isomorphism $K \mathcal{S}_{q}(n, r) \rightarrow \mathcal{J}(n, r)_{K}$ when tensored with the field of fractions $K$ of $A$.

Proof. See [7, 2.3]. The latter equation holds, since $f_{\underline{a}, \underline{b}, \underline{d}}=0$ unless $\underline{d} \leq_{L} \underline{b}$, and Q9 implies $\underline{d} \sim_{L} \underline{b}$ in this case. Also, we can safely sum over all of $\mathcal{D}(n, r)$ neglecting the index $\mu$, since all elements $\underline{d} \in \mathcal{D}(n, r)$ fulfill $r o(\underline{d})=c o(\underline{d})$ by definition (see Definition 2.5 and the remark there) and $f_{\underline{a}, \underline{d}, \underline{b}}=0$ unless $c o(\underline{a})=\operatorname{ro}(\underline{d})$ anyway.

We can now present our main theorem, which links our Wedderburn basis $\mathcal{B}$ to the asymptotic algebra:

Theorem 5.4 (Preimage of the $t$-basis under the Du-Lusztig homomorphism). Let $\tau$ be an arbitrary symmetrising trace form. All dual bases in the following are meant with respect to $\tau$.

With the above notation we have

$$
\Phi\left(c_{\underline{d}}^{-1} \theta_{\underline{c}} \theta_{\underline{d}}^{\vee}\right)=t_{\underline{c}} \quad \text { for all } \underline{c} \in M(n, r) .
$$

Proof. The rightmost sum in Theorem 5.3 has the advantage that it provides a formula for the image of an arbitrary element $h \in K_{\mathcal{S}}(n, r)$ under the Du-Lusztig homomorphism, since it is obviously $K$-linear in $\theta_{\underline{a}}$ :

$$
\Phi(h)=\sum_{\substack{b \in M(n, r) \\ \bar{b} \in \mathcal{D}(n, r) \\ \underline{\bar{d}^{\prime} \in \mathcal{D}(n)}}} \tau\left(h \cdot \theta_{{\underline{d^{\prime}}}^{\prime}} \theta_{\underline{b}}^{\vee}\right) \cdot t_{\underline{b}}
$$


(recall $\left.\tau\left(\theta_{\underline{a}} \theta_{\underline{d}^{\prime}} \theta_{b}^{\vee}\right)=f_{\underline{a}, \underline{d^{\prime}}, \underline{b}}\right)$. But now we can immediately set $h:=c_{\underline{d}}^{-1} \theta_{c} \theta_{d}^{\vee}$ for some $\underline{c} \in M(n, r)$ and $\underline{d} \in \mathcal{D}(n, r)$ with $\underline{c} \sim_{L} \underline{d}$. The value $\tau\left(c_{\underline{d}}^{-1} \theta_{\underline{c}} \theta_{\underline{d}}^{\vee} \cdot \theta_{\underline{d^{\prime}}} \theta_{\underline{b}}^{\vee}\right)$ is zero (see Lemma 4.2) unless $\underline{b} \leq_{R} \underline{c} \sim_{L} \underline{d} \leq_{R} \underline{d^{\prime}} \sim_{L} \underline{b}$ and this implies $\underline{b} \sim_{R} c$ and $\underline{d^{\prime}} \sim_{R} \underline{d}$ using Q4 and Q10; but this means $\underline{d^{\prime}}=\underline{d}$ by Q13 and the definition of $\sim_{R}$ and thus $\underline{b}=\underline{c}$ because of Lemma 3.8 Thus, in the sum there is only one non-zero summand, which is $\tau\left(c_{\underline{d}}^{-1} \theta_{\underline{c}} \theta_{\underline{d}}^{\vee} \cdot \theta_{\underline{d}} \theta_{\underline{c}}^{\vee}\right) t_{\underline{c}}$. Now everything is in a single left cell such that we can use Theorem 4.5 to get

$$
\tau\left(c_{\underline{d}}^{-1} \theta_{\underline{c}} \theta_{\underline{d}}^{\vee} \cdot \theta_{\underline{d}} \theta_{\underline{c}}^{\vee}\right) \cdot t_{\underline{c}}=\tau\left(\theta_{\underline{c}} \theta_{\underline{c}}^{\vee}\right) \cdot t_{\underline{c}}=t_{\underline{c}}
$$

as claimed.

We can summarise our results in the following way:

Theorem 5.5 (New interpretation of the Du-Lusztig homomorphism). Let $\tau$ be an arbitrary symmetrising trace form on $K \mathcal{S}_{q}(n, r)$. We define the set $\mathcal{B}$ as in Theorem 4.12 and we set

$$
\mathcal{J}_{\tau}=\langle\mathcal{B}\rangle_{A}
$$

The following diagram commutes and all unmarked arrows are identities or natural inclusions:

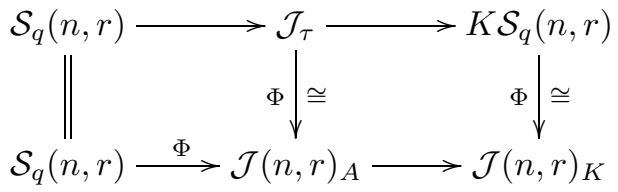

Thus, the asymptotic algebra $\mathcal{J}(n, r)_{A}$ is nothing but the A-span of our Wedderburn basis and the Du-Lusztig homomorphism $\Phi$ can simply be interpreted as the inclusion of $\mathcal{S}_{q}(n, r)$ into $\langle\mathcal{B}\rangle_{A}$. Furthermore, our results directly and explicitly show that $\langle\mathcal{B}\rangle_{A}$ is isomorphic as an A-algebra to a direct sum of full matrix rings over A.

\section{A hypothesis ImPLying James' CONJECTURE}

In this section we show how our results provide an equivalent formulation of a conjecture about the representation theory of specialisations of the $q$-Schur algebra. We first recall the conjecture.

The construction of the Iwahori-Hecke algebra of type A and of the $q$-Schur algebra as in Section 2 together with their Kazhdan-Lusztig bases can be carried out over an arbitrary integral domain $R$ with quotient field $k$ and with an arbitrary invertible parameter $q \in R$ having a square root in that domain. We denote the resulting algebra by $\mathcal{S}_{q}(n, r)_{R}$ and its extension of scalars to $k$ by $\mathcal{S}_{q}(n, r)_{k}$.

The case of the Laurent polynomial ring $A=\mathbb{Z}\left[v, v^{-1}\right]$ and $q=v^{2}$ is called the "generic" case, since for every other choice $(R, q)$ there is a ring homomorphism $\varphi: \mathbb{Z}\left[v, v^{-1}\right] \rightarrow R$ mapping $v^{2}$ to $q \in R$, which induces a ring homomorphism $\mathcal{S}_{v^{2}}(n, r)_{A} \rightarrow \mathcal{S}_{q}(n, r)_{R} \subseteq \mathcal{S}_{q}(n, r)_{k}$. This is called a "specialisation".

It is known, that $\mathcal{S}_{q}(n, r)_{k}$ is semisimple unless $q$ is an $e$-th root of unity. If $q$ is a root of unity, then there is a decomposition matrix, which records the multiplicities of the simple modules in the so-called "standard modules". For the case that $k$ has characteristic zero, recent work by Lascoux, Leclerc and Thibon, and Varagnolo and Vasserot yields a complete determination of these decomposition matrices (see 
[18, 10] and the references there). However, the case of positive characteristic is still open.

James' conjecture is a statement about this modular case (see [13, §4] and [10, $\S 3])$. Roughly speaking, it asserts that if $k$ is a field of characteristic $\ell$ and $e$ is the multiplicative order of the parameter $q \in k$ and $\ell$ is greater than $r$, then the decomposition matrix of $\mathcal{S}_{q}(n, r)_{k}$ does not depend on the particular value of $\ell$ but only on $e$. Note that James originally used the condition $\ell e>r$ which is a bit weaker, but the important point is that the condition holds for all but finitely many values of $\ell$.

We now want to make this statement more precise. Both the simple modules and the standard modules have a labelling by the set $\Lambda^{+}(n, r)$ or partitions of $r$ with at most $n$ parts. Let $V_{k, q}^{\lambda}$ denote the standard module and $M_{k, q}^{\mu}$ the simple module of $\mathcal{S}_{q}(n, r)_{k}$ corresponding to $\lambda$ and $\mu$, respectively. Then the decomposition matrix for $\mathcal{S}_{q}(n, r)_{k}$ consists of the numbers

$$
d_{\lambda, \mu}^{k, q}:=\text { multiplicity of } M_{k, q}^{\mu} \text { in } V_{k, q}^{\lambda} .
$$

Conjecture 6.1 (James, see [13, §4] and [10, §3]). If $\ell>r$ and $e$ is the multiplicative order of $q \in k$, then $d_{\lambda, \mu}^{k, q}=d_{\lambda, \mu}^{\mathbb{Q}\left(\zeta_{e}\right), \zeta_{e}}$ for all $\lambda, \mu \in \Lambda(n, r)$, where $\zeta_{e}$ is a complex primitive e-th root of unity.

Meinolf Geck has shown in [11, Theorem 1.2] that this statement is equivalent to the fact that, for $\ell>r$, the rank of the Du-Lusztig homomorphism $\Phi$ : $\mathcal{S}_{q}(n, r)_{k} \rightarrow \mathcal{J}(n, r)_{k}$ with respect to the two bases $\left(\theta_{\underline{a}}\right)_{\underline{a} \in M(n, r)}$ and $\left(t_{\underline{a}}\right)_{\underline{a} \in M(n, r)}$ is equal to the rank of the corresponding Du-Lusztig homomorphism $\mathcal{S}_{\zeta_{2 e}^{2}}(n, r)_{\mathbb{Q}\left(\zeta_{2 e}\right)} \rightarrow$ $\mathcal{J}(n, r)_{\mathbb{Q}\left(\zeta_{2 e}\right)}$ with respect to the corresponding bases, where $e$ is the multiplicative order of $q \in k$ and $\zeta_{2 e}$ is a primitive $2 e$-th root of unity in $\mathbb{C}$. In particular, the rank does not depend on the characteristic $\ell$ of $k$.

In view of our Theorem 5.5 this immediately implies:

Theorem 6.2 (An equivalent formulation of James' conjecture). Let $\left\{\theta_{\underline{a}} \mid \underline{a} \in\right.$ $M(n, r)\}$ be the Du-Kazhdan-Lusztig basis of $\mathcal{S}_{q}(n, r)$, let $\tau$ be a symmetrising trace form for $K \mathcal{S}_{q}(n, r)$ in the sense of Section 4 and $\left\{c_{\underline{d}}\right\}_{\underline{d} \in \mathcal{D}(n, r)}$ the corresponding Schur elements as above. Let $\left\{\theta_{\underline{a}}^{\vee} \mid \underline{a} \in M(n, r)\right\}$ be the dual basis of $\left\{\theta_{\underline{a}} \mid \underline{a} \in\right.$ $M(n, r)\}$ with respect to $\tau$. Let $\overline{\mathcal{B}}$ be the basis defined in Theorem 4.12, Let $s:=$ $|M(n, r)|$ and $M=\left(m_{\underline{a}, \underline{b}}\right)_{\underline{a}, \underline{b} \in M(n, r)} \in A^{s \times s}$ be the matrix, for which

$$
\theta_{\underline{a}}=\sum_{\underline{c} \in M(n, r)} m_{\underline{a}, \underline{c}} \cdot c_{\underline{d}}^{-1} \theta_{\underline{c}} \theta_{\underline{d}}^{\vee}
$$

with $c_{\underline{d}}^{-1} \theta_{\underline{c}} \theta_{\underline{d}}^{\vee} \in \mathcal{B}$ holds for all $\underline{a} \in M(n, r)$.

Let $\ell$ be a prime and $\varphi_{\ell}: \mathbb{Z}\left[v, v^{-1}\right] \rightarrow \mathbb{F}_{\ell}$ a ring homomorphism, such that the multiplicative order of $\varphi_{\ell}(v)$ is equal to $2 e$. Denote by $\varphi_{\ell}(M)$ the matrix in $\mathbb{F}_{\ell}^{s \times s}$ that one gets by applying the ring homomorphism $\varphi_{\ell}$ to every entry of $M$.

Let $\zeta_{2 e}$ be a primitive $2 e$-th root of unity in $\mathbb{C}$ and $\varphi_{e}: \mathbb{Z}\left[v, v^{-1}\right] \rightarrow \mathbb{Q}\left(\zeta_{2 e}\right)$ be the ring homomorphism mapping $v$ to $\zeta_{2 e}$. Then there is a ring homomorphism $\varphi_{\ell}^{e}: \mathbb{Z}\left[\zeta_{2 e}\right] \rightarrow \mathbb{F}_{\ell}$ with $\varphi_{\ell}=\varphi_{\ell}^{e} \circ \varphi_{e}$. Denote by $\varphi_{e}(M)$ the matrix in $\mathbb{Q}\left(\zeta_{2 e}\right)^{s \times s}$ that one gets by applying the ring homomorphism $\varphi_{e}$ to every entry of $M$.

Then James' conjecture is equivalent to the fact that for $\ell>r$ the ranks of $\varphi(M)$ (over $\mathbb{F}_{\ell}$ ) and of $\varphi_{e}(M)$ (over $\mathbb{Q}\left(\zeta_{2 e}\right)$ ) are equal. 
Let $\tau$ be a symmetrising trace form on $K \mathcal{S}_{q}(n, r)$. We denote by $\left\{\theta_{\underline{a}} \mid \underline{a} \in\right.$ $M(n, r)\}$ the Du-Kazhdan-Lusztig basis of $\mathcal{S}_{q}(n, r)$ and by $\left\{\theta_{a}^{\vee} \mid \underline{a} \in M(n, r)\right\}$ its dual basis relative to $\tau$. As above, we denote by $\mathcal{B}$ the Wedderburn basis obtained in Theorem 4.12. Moreover, we denote by $M=\left(m_{\underline{a}, \underline{b}}\right)_{\underline{a}, \underline{b} \in M(n, r)}$ the change of basis matrix from $\left\{\theta_{\underline{a}} \mid \underline{a} \in M(n, r)\right\}$ to $\mathcal{B}$ as above and by $P_{\tau}=\left(p_{\underline{a}, \underline{b}}\right)_{\underline{a}, \underline{b} \in M(n, r)}$ the change of basis matrix from $\left\{\theta_{\underline{a}} \mid \underline{a} \in M(n, r)\right\}$ to $\left\{\theta_{\underline{a}}^{\vee} \mid \underline{a} \in M(n, r)\right\}$, that is,

$$
\theta_{\underline{a}}=\sum_{\underline{b} \in M(n, r)} p_{\underline{a}, \underline{b}} \cdot \theta_{\underline{b}}^{\vee}
$$

for all $\underline{a} \in M(n, r)$. Formula (4.2) implies that

$$
P_{\tau}=\left(\tau\left(\theta_{\underline{a}} \theta_{\underline{b}}\right)\right)_{\underline{a}, \underline{b} \in M(n, r)} \quad \text { and } \quad P_{\tau}^{-1}=\left(\tau\left(\theta_{\underline{a}}^{\vee} \theta_{\underline{b}}^{\vee}\right)\right)_{\underline{a}, \underline{b} \in M(n, r)} .
$$

Lemma 6.3. With the above notation, the matrix

$$
D=M^{T} P_{\tau}^{-1} M
$$

is monomial and its entries are the Schur elements $c_{\underline{d}}$ associated to $\underline{d} \in \mathcal{D}(n, r)$ as in Definition 4.11.

Proof. The matrix $M^{T}$ is the change of basis matrix from $\mathcal{B}^{\vee}$ to $\left\{\theta_{a}^{\vee} \mid \underline{a} \in M(n, r)\right\}$ and thus the matrix $D$ is the change of basis matrix from $\mathcal{B}^{\vee}$ to $\mathcal{B}$, that is,

$$
\theta_{\underline{c}} \theta_{\underline{d}}^{\vee}=\sum_{\underline{c^{\prime}} \in M(n, r)} d_{\underline{c}, \underline{c}^{\prime}}{\underline{\underline{d^{\prime}}}}^{-1} \theta_{\underline{c}^{\prime}} \theta_{\underline{d}^{\prime}}^{\vee}
$$

for all $\theta_{\underline{c}} \theta_{\underline{d}}^{\vee} \in \mathcal{B}^{\vee}$. Using Proposition 4.16, the result follows.

Proposition 6.4 (A hypothesis implying James' conjecture). Let $\tau$ be a symmetrising trace form on $K \mathcal{S}_{q}(n, r)$ in the sense of Section 4 and $\left\{c_{\underline{d}}\right\}_{\underline{d} \in \mathcal{D}(n, r)}$ the corresponding Schur elements as above. Let $\varphi_{e}: A \rightarrow \mathbb{Z}\left[\zeta_{2 e}\right], v \mapsto \zeta_{2 e}$ be a specialisation to characteristic 0 where $v^{2}$ is mapped to a primitive e-th root of unity in a cyclotomic field and $\varphi_{\ell}: A \rightarrow \mathbb{F}_{\ell}$ is a second specialisation to characteristic $\ell$ such that there is a ring homomorphism $\varphi_{\ell}^{e}: \mathbb{Z}\left[\zeta_{2 e}\right] \rightarrow \mathbb{F}_{\ell}$ with $\varphi_{\ell}=\varphi_{\ell}^{e} \circ \varphi_{e}$. We suppose that $\ell>r$ and the following hypotheses on $\tau$ :

- The Schur elements $c_{\underline{d}}$ for $\underline{d} \in \mathcal{D}(n, r)$ lie in $A$.

- The coefficients of the matrix $P_{\tau}^{-1}$ lie in $A$.

- Let a be the number of Schur elements $c_{\underline{d}}$ for $\underline{d} \in \mathcal{D}(n, r)$ that do not vanish under $\varphi_{e}$ and $b$ the number of Schur elements that do not vanish under $\varphi_{\ell}$. The numbers $a$ and $b$ are both equal to the rank over $\mathbb{Q}\left(\zeta_{2 e}\right)$ of the matrix $\varphi_{e}(M)$ for $M$ from above.

Note that we denote with the notation $\varphi_{e}(M)$ the matrix one gets from $M$ by applying the ring homomorphism $\varphi_{e}$ on every entry.

If $\tau$ can be found by fulfilling all these hypotheses, then James' conjecture holds for all $\ell>r$ for which $\varphi_{\ell}$ as above exist.

Proof. We denote by $M$ the change of basis matrix from $\left\{\theta_{a} \mid \underline{a} \in M(n, r)\right\}$ to $\mathcal{B}$ as above. Then Lemma 6.3 asserts that

$$
D=M^{T} P_{\tau}^{-1} M .
$$

Thanks to Theorem 4.12, the coefficients of the matrix $M$ lie in $A$. By hypothesis, the matrix $P_{\tau}^{-1}$ has coefficients in $A$. By Lemma 6.3 and the first hypothesis the entries of $D$ are also in $A$. 
Since the matrices $D, M, M^{T}$, and $P_{\tau}^{-1}$ have coefficients in $A$, the matrices $\varphi_{e}(D), \varphi_{e}(M), \varphi_{\ell}(D), \varphi_{\ell}(M), \varphi_{\ell}\left(M^{T}\right)$ and $\varphi_{\ell}\left(P_{\tau}^{-1}\right)$ are well defined. We then have the equality

$$
\varphi_{\ell}(D)=\varphi_{\ell}\left(M^{T}\right) \cdot \varphi_{\ell}\left(P_{\tau}^{-1}\right) \cdot \varphi_{\ell}(M),
$$

implying that $\operatorname{rk}_{\mathbb{F}_{\ell}}\left(\varphi_{\ell}(D)\right) \leq \operatorname{rk}_{\mathbb{F}_{\ell}}\left(\varphi_{\ell}(M)\right)$. Moreover, we have $\varphi_{\ell}(M)=\varphi_{\ell}^{e}\left(\varphi_{e}(M)\right)$. Since $\varphi_{\ell}^{e}$ is a ring homomorphism, we deduce that

$$
\operatorname{rk}_{\mathbb{F}_{\ell}}\left(\varphi_{\ell}(M)\right) \leq \operatorname{rk}_{\mathbb{Q}\left(\zeta_{2 e}\right)}\left(\varphi_{e}(M)\right) .
$$

Since $D$ is a monomial matrix containing only the Schur elements as non-zero entries, the numbers $a$ and $b$ from the hypotheses are the ranks of $\varphi_{e}(D)$ and $\varphi_{\ell}(D)$, respectively. However, if as in the last hypothesis the ranks of $\varphi_{e}(M)$ and $\varphi_{\ell}(D)$ are equal, then it follows that $\operatorname{rk}_{\mathbb{F}_{\ell}}\left(\varphi_{\ell}(M)\right) \leq \operatorname{rk}_{\mathbb{F}_{\ell}}\left(\varphi_{\ell}(D)\right)$. We then deduce that

$$
\operatorname{rk}_{\mathbb{F}_{\ell}}\left(\varphi_{\ell}(M)\right)=\operatorname{rk}_{\mathbb{F}_{\ell}}\left(\varphi_{\ell}(D)\right),
$$

and the result now follows from Theorem 6.2

Remark 6.5. To prove James' conjecture it is enough to find a symmetrising trace form $\tau$ on $K \mathcal{S}_{q}(n, r)$ such that the hypotheses of Proposition 6.4 are satisfied. We notice that the assumption on $P_{\tau}$ in the statement of Proposition 6.4 is "generic" in the sense that this property only depends on the "generic" $q$-Schur algebra, but not on specialisations over finite fields.

Remark 6.6. We can replace the second assumption of Proposition 6.4 by the fact that the matrix $P_{\tau}^{-1} M$ (or $M^{T} P_{\tau}^{-1}$ ) has its coefficients in $A$.

Remark 6.7. For the usual trace form $\tau_{0}$ on Iwahori-Hecke algebras of type $A$, we note that the assumptions of Proposition 6.4 hold. Then using [16], we can prove in a way similar to the one of the proof of Proposition 6.4 that the rank of the Lusztig homomorphism (specialised in a finite field $\mathbb{F}_{\ell}$ by $\varphi_{\ell}: A \rightarrow \mathbb{F}_{\ell}$ mapping $v^{2}$ to an element $q \in \mathbb{F}_{\ell}$ with multiplicative order $e$ as above) does not depend on $\ell$. However, as noted by Geck in [11] an analogue result such as Theorem 6.2 in Iwahori-Hecke algebras does not imply the Iwahori-Hecke algebras of James' conjecture.

\section{ACKNOWLEDGEMENT}

We would like to thank the referee for valuable comments to correct and improve this paper.

\section{REFERENCES}

[1] Charles W. Curtis and Irving Reiner. Methods of representation theory. Vol. I. Wiley Classics Library. John Wiley \& Sons Inc., New York, 1990. With applications to finite groups and orders, Reprint of the 1981 original, A Wiley-Interscience Publication. MR.1038525 (90k:20001)

[2] Richard Dipper and Gordon James. Representations of Hecke algebras of general linear groups. Proc. London Math. Soc. (3), 52(1):20-52, 1986. MR812444 (88b:20065)

[3] Richard Dipper and Gordon James. The $q$-Schur algebra. Proc. London Math. Soc. (3), 59(1):23-50, 1989. MR997250 (90g:16026)

[4] Richard Dipper and Gordon James. $q$-tensor space and $q$-Weyl modules. Trans. Amer. Math. Soc., 327(1):251-282, 1991. MR:1012527 (91m:20061)

[5] Jie Du. Kazhdan-Lusztig bases and isomorphism theorems for $q$-Schur algebras. In KazhdanLusztig theory and related topics (Chicago, IL, 1989), volume 139 of Contemp. Math., pages 121-140. Amer. Math. Soc., Providence, RI, 1992. MR1197832 (94b:17019)

[6] Jie Du. Canonical bases for irreducible representations of quantum $\mathrm{GL}_{n}$. II. J. London Math. Soc. (2), 51(3):461-470, 1995. MR1332884 (96h:17016) 
[7] Jie Du. q-Schur algebras, asymptotic forms, and quantum $\mathrm{SL}_{n}$. J. Algebra, 177(2):385-408, 1995. MR1355207(96k:17021)

[8] Jie Du. Cells in certain sets of matrices. Tohoku Math. J. (2), 48(3):417-427, 1996. MR:1404511 (97e:20012)

[9] Jie Du, Brian Parshall, and Leonard Scott. Quantum Weyl reciprocity and tilting modules. Comm. Math. Phys., 195(2):321-352, 1998. MR1637785 (99k:17026)

[10] Meinolf Geck. Representations of Hecke algebras at roots of unity. Astérisque, (252):Exp. No. 836, 3, 33-55, 1998. Séminaire Bourbaki. Vol. 1997/98. MR1685620 (2000g:20018)

[11] Meinolf Geck. Kazhdan-Lusztig cells, $q$-Schur algebras and James' conjecture. J. London Math. Soc. (2), 63(2):336-352, 2001. MR1810133(2002g:20028)

[12] Meinolf Geck and Götz Pfeiffer. Characters of Finite Coxeter Groups and Iwahori-Hecke Algebras, volume 21 of London Mathematic Society, New Series. Oxford University Press, Oxford, 2000. MR $1778802(2002 \mathrm{k}: 20017)$

[13] Gordon James. The decomposition matrices of $\mathrm{GL}_{n}(q)$ for $n \leq 10$. Proc. London Math. Soc. (3), 60(2):225-265, 1990. MR 1031453 (91c:20024)

[14] David Kazhdan and George Lusztig. Representations of Coxeter groups and Hecke algebras. Invent. Math., 53(2):165-184, 1979. MR.560412 (81j:20066)

[15] George Lusztig. Hecke algebras with unequal parameters, volume 18 of CRM Monograph Series. American Mathematical Society, Providence, RI, 2003. MR.1974442 (2004k:20011)

[16] Max Neunhöffer. Kazhdan-Lusztig basis, Wedderburn decomposition, and Lusztig's homomorphism for Iwahori-Hecke algebras. J. Algebra, 303(1):430-446, 2006. MR.2253671 (2008a:20012)

[17] Jian-Yi Shi. The Kazhdan-Lusztig Cells in Certain Affine Weyl Groups, volume 1179 of Lecture Notes in Mathematics. Springer-Verlag, Berlin, Heidelberg, New York, Tokyo, 1986. MR835214 (87i:20074)

[18] Michela Varagnolo and Eric Vasserot. On the decomposition matrices of the quantized Schur algebra. Duke Math. J., 100(2):267-297, 1999. MR1722955 (2001c:17029)

Ruhr-Universität Bochum, Fakultät für Mathematik, D-44780 Bochum, Germany Current address: Institut de Mathèmatiques de Jussieu, UFR de Mathèmatiques, 175, rue du Chevaleret, F-75013 Paris

E-mail address: brunat@math.jussieu.fr

School of Mathematics and Statistics, Mathematical Institute, North Haugh, St Andrews, Fife KY16 9SS, Scotland, United Kingdom

E-mail address: neunhoef@mcs.st-and.ac.uk 